\title{
The Impact of Auditing Strategies on Insurers' Profitability
}

\begin{abstract}
Insurance claims fraud is a major concern in the insurance industry. In this paper, we develop a model framework based on a costly state verification setting in which - while policyholders observe the amount of loss privately - the insurance company can decide to audit incoming claims at some cost. The aim is to derive optimal auditing strategies from the insurance company perspective while maintaining contract attractiveness to policyholders who are willing to adhere to the insurance relationship. The central outcome of the model is an auditing range which selects those claims which should be subject to verification. In extension of the existing body of knowledge in the area of costly state verification, we take account of the possibility for each stakeholder to adapt its behavioral strategy over the course of several periods. By this means, we take into consideration that changes in policyholder defrauding behavior over time can have a crucial impact on the optimal auditing strategy and vice versa. Using a numerical approach based on Monte Carlo simulations, we illustrate and analyze the impact of different parameterizations on the optimal auditing range by means of a sensitivity analysis.
\end{abstract}

\section{Introduction}

Insurance claims fraud is one of the major industry concerns. It occurs in all classes of insurance and accounts for a substantial portion of indemnity payments each year, yet due to its nature, it is difficult to estimate the total value. The Insurance Research Council (2008) estimated excess payments due to fraudulent claims in 2007 as somewhere between $\$ 4.8$ and $\$ 6.8$ billion in the auto injury insurance sector in the U.S. alone, corresponding to 13 to 18 percent of total payments. These fraudulent activities, while undertaken by some individuals, have an impact on the policyholder population as a whole through higher insurance premiums (see Tennyson (2008)).

Previous research has explained the existence of fraud due to information being asymmetrically distributed between policyholder and the corresponding insurance company (see, e.g., Derrig (2002)). Since the insured may hold private information about the actual amount of the loss suffered, there is a possibility of misrepresentation. Based on the approach of costly state verification (see, e.g., Townsend (1979), Mookherjee and Png (1989), Bond and Crocker (1997), Picard and Fagart (1999), Picard (2000), Viaene, Ayuso, Guillen, Van Gheel, and Dedene (2007)), the insurance company can consistently choose to audit incoming claims in order to determine their truthfulness. In cases when fraudulent activities 
have been proven, a penalty payment can be imposed on the policyholder. However, these verification processes incur costs. From the insurance company perspective this implies that the costs for auditing have to be traded off against the savings resulting from detected fraud. One of the costs may be policyholder attitude, and therefore, the policyholder's point of view needs to be considered as well. According to Viaene and Dedene (2004), individuals are more likely to develop an opportunistic attitude towards insurance fraud after having negative experiences with their insurance companies. Underpaid claims or long waiting periods for indemnity payments may encourage fraudulent behavior in the future.

An opposing strategy to minimize the occurrence of insurance fraud involves the implementation of bonus-malus systems. Dionne and Vanasse (1992) and Moreno, Vázquez, and Watt (2006) develop model frameworks where, instead of performing costly verification processes, the policyholder's insurance premium is increased whenever he or she files a claim in the previous period. This approach is applicable in the case of insurance contract renewals; however, in a few countries policyholders may avoid this penalization mechanism by switching their insurance company (see, e.g., Dionne and Ghali (2005)).

An additional strand of research dealing with the occurrence and handling of insurance claims fraud is based on the costly state falsification approach introduced by Lacker and Weinberg (1989) and adapted to the insurance setting by Crocker and Morgan (1998) and Crocker and Tennyson (2002). While the premise with regard to the asymmetric distribution of information remains unchanged, this time the policyholder engages in costly manipulations such that a verification of the claims becomes impossible.

The term fraud has a rather negative connotation implying the engagement in illegal activities such as staged accidents. The range of actions, however, which is colloquially subsumed under this notion, is far broader (see, e.g., Picard (2001), Derrig (2002), Tennyson (2008)). In general, a distinction is made between ex-ante and ex-post moral hazard, referring to the timing of fraudulent behavior. The former occurs by the time of insurance purchase, e.g., if the potential policyholder fails to provide relevant information which might have resulted in unfavorable contract conditions or even in a rejection by the insurance company (see, e.g., Picard (2001)). The term ex-post moral hazard implies the engagement in fraudulent activities by the time of claims filing, i.e., after a potential loss has been suffered. In this context, there are several possible distinctions which can be made. A common one is to differ between criminal fraud, also called hard fraud, and soft fraud which is located in an ethical gray area (see, e.g., Tennyson (2008)). Derrig (2002) defines criminal fraud as 'the willful act of obtaining money or value from an insurer under false pretenses or material misrepresentations'. While it is assumed that only a small number of claims involve outright fraud, the more frequent and more costly type of fraudulent behavior falls under soft fraud (see, e.g., Weisberg and Derrig (1991), Viaene and Dedene (2004), Tennyson (2008)). Even though there is not an explicit definition of the term, it is associated with the misrepresentation of the loss magnitude after its occurrence, namely that claimants exaggerate the amount to obtain higher indemnity payments. A notion often used in this context is claim inflation or buildup. In this paper, we will refer to fraud as soft fraud or buildup, that is we assume that some policyholders inflate the magnitude of loss after its occurrence if it appears profitable. We suppose that there is no opportunity for false claims and only allow for false claim amounts.

In addition to the policyholders, third parties like service providers might also be involved in fraudulent activities to exaggerate the loss amount (see, e.g., Tennyson (2008), Dulleck and Kerschbamer 
(2006) Derrig and Zicko (2002)). Prominent examples are available in the automobile insurance sector. Derrig and Zicko (2002) found that repair shops are likely to have developed insight about the insurance companies' prevalent auditing strategies due to repeated experiences in repairing cars for insured damages. As a consequence, it is possible to adjust the defrauding strategy. Such actions are either undertaken without the knowledge of the policyholder, or performed by mutual agreement between the policyholder and the corresponding repair shop. The service providers' incentive to engage in fraudulent activities against the insurance company may stem from the hope to gain the policyholders' favor. As a result, the latter might request their services again at a later point in time. For the purpose of our study, we will assume that all fraudulent activities - even if committed by repair shops - are advantageous for the policyholders only.

Considering its great prevalence, effective measures in dealing with the phenomenon of insurance claims fraud need to be found. Many insurers have dedicated teams to identify and combat fraudulent claims. Morley, Ball, and Ormerod (2006) as well as Viaene, Ayuso, Guillen, Van Gheel, and Dedene (2007) find that verification processes are initiated whenever investigators detect anomalies or inconsistencies in the circumstances of the loss event or unusual behavior by the claimant. State-of-the art classification techniques for fraud detection in the automobile insurance sector are discussed for example by Viaene, Derrig, Baesens, and Dedene (2002), whereby predictive analytics and Bayesian learning techniques are contrasted. More recently, the study by Bearing Point (2008) analyzes the automation levels in fraud detection, and Mahlow, Maier, Müller, Schmidt, and Wagner (2015) stress the importance of using all available statistical data. Using logistic regression on a comprehensive data set, Müller (2014) shows in an empirical analysis how determinants for fraud behavior can be identified.

This approach seems to be suitable for filed claims that exhibit obvious signs of potential fraudulent behavior and/or those of significant magnitude. Apparently, high-magnitude claims are subject to verification right away. The majority of claims, however, appear to be legitimate at first sight, seeking low to medium indemnity payments, which do not trigger immediate investigation. Consequently, the key question is how to deal with the fraudulent cases that appear legitimate and that account for the majority of inappropriate indemnity payments (see, e.g., Derrig (2002)).

We therefore focus on developing audit strategies which help to improve the efficiency of the claims settlement process with regard to inconspicuous claims. The key element in our model framework - which pursues the costly state verification approach - is the use of threshold values which indicate whether an incoming claim should be verified or not. This approach allows the insurer to determine the optimal auditing strategy based on the magnitude of the filed claim. For this purpose, we make use of information on policyholder claiming and, more importantly, defrauding behavior which insurance companies are expected to maintain due to previous experiences and verification processes. In particular, the percentage of fraudprone policyholders among the population has an impact on the actual threshold values for auditing. Interviews with experts as well as previous research (see, e.g., Belhadji, Dionne, and Tarkhani (2000), Viaene, Ayuso, Guillen, Van Gheel, and Dedene (2007), Bermúdez, Pérez, Ayuso, Gómez, and Vázquez (2008)) have shown that numerous criteria exist which help indicate the potential fraud behavior of an individual. Such factors may include gender, nationality or place of residence. Additionally, investigations in the past have shown that certain accidents are more prone to fraud than others. For example, 
wind screen and glass damage as well as thefts from the vehicle interior are known to be cases where policyholders are likely to report exaggerated claim amounts. Combining this information on individual characteristics with fraud signals presented by Dionne, Giuliano, and Picard (2009)), one obtains accurate estimators for the share of defrauders among claimants.

In what follows, a model framework is presented based on a costly state verification setting in which - while policyholders observe the amount of loss privately - the insurance company can decide to audit incoming claims at some cost. Our goal is to derive optimal auditing strategies from the insurance company perspective indicating which of the incoming claims are subject to verification. In any case, we take into account that contract conditions must still be attractive to policyholders such that they are willing to adhere to the insurance relationship. A key element of our study is the option of both stakeholders adapting their behavior accordingly based on different signals. Subsequently, the impact of the optimal auditing strategy on insurers' profitability is analyzed.

Unlike other strands of research in this area (e.g., Townsend (1979), Picard and Fagart (1999)), which often set up the problem of optimizing insurance contracts in a way such that policyholders always report loss amounts truthfully in the equilibrium state, we do not develop an auditing scheme which completely prevents or deters insurance claims fraud. From a macroeconomic point of view, both the approach and the result are desirable. From a single company perspective, however, this does not necessarily hold true. Similarly as with Dionne, Giuliano, and Picard (2009), our aim is to minimize the insurer's overall costs from fraud, in general, including paying some portion of fraudulent claims, as well as incurring expenses to detect and address fraud, and in experiencing the reputational effects of investigations themselves. In fact, we can show that for low costs per audit process the insurance company's net present value increases in the presence of fraud compared to the case where no fraud exists. Our findings are in line with the assumption made by Watt (2003).

Allowing for the existence of some fraudulent activities accounts for the widespread attitude of considering insurance as an investment which is expected to yield a return. As evidence, a study from GDV (2011) reported that more than $20 \%$ of Germans consider insurance fraud to be a 'gentlemen's offense' which is committed by almost everyone at least once. This point of view can be found among all socio-demographic groups. Duffield and Grabosky (2001) compile different approaches individuals use to justify fraud. Among others, insurance fraud is assumed to cause no significant harm; insurers are accepted targets which can afford it; buildup is a way to recover past premium payments (see also Miyazaki (2008)). Given this line of reasoning, we assume therefore that there will always be policyholders trying to defraud by inflating their magnitude of loss. In this context, auditing processes can help to minimize the share of fraudulent activities among the policyholder population. But a complete eradication seems unlikely.

Including the option to change one's strategy based on signals provides another insightful result. We can show that there are situations where - while the ability to adapt one's behavior might be desirable for the insurance company - this option is disadvantageous from the policyholder perspective. This observation stands in contrast to the widespread opinion that the adaptation of the defrauding strategy, especially based on signals from service providers, would be favorable from the individual's point of view. 
In our model, the policyholders and service providers which might be involved may obtain signals and information on the basis of which they change the defrauding strategy, however, they do not know the exact auditing threshold values applied by the insurer nor do they have enough information to derive them themselves. This is a crucial assumption in our model which is based on real world observations. Leaving out this assumption, any auditing strategy would be redundant since the policyholder would know how to adapt his fraud behavior in such a way as to avoid being caught. From the insurance company perspective, it would not make sense to verify any incoming claim in this case.

The remainder of this paper is organized as follows: We start by presenting the model framework and optimization problem in Section 2. Section 3 constitutes the introduction of the policyholder's and insurer's respective strategies as well as the behavioral adaptation process. The corresponding numerical results are presented in Section 4. In Section 5, we analyze practical implications, before we conclude in Section 6.

\section{Model Framework and Stakeholders' Positions}

In a first step, we describe in Section 2.1 basic assumptions about the portfolio structure and the filing and handling process of the claims within the portfolio. In a second step, we introduce each contract's contribution margin and the insurer's net present value (Section 2.2). Finally, the policyholder wealth positions and their expected utility is analyzed (Section 2.3).

\subsection{Basic Assumptions about the Filing and Handling of Claims}

We consider an insurance company with a fixed number of policyholders belonging to a homogeneous portfolio. The latter are assumed to differ from one another only in their willingness to defraud, i.e., the population consists of both honest individuals who never commit fraud and those who defraud if it appears to be profitable. We assume the percentage of fraud-prone policyholders among the population to be $p$. Depending on the loss amount and the prevalent defrauding strategy, fraud-prone policyholders may or may not defraud, so that the percentage of claims which actually contain buildup might be lower.

Since we assume all individuals belong to the existing homogeneous policyholder population, they all pay a constant insurance premium $P$ at the beginning of a period. We assume this premium equals the given market premium which is charged by other insurance companies as well. At the same time, with probability $0 \leq \pi \leq 1$, they face some uncertain loss $\theta$ of a stochastic amount which, by the time of occurrence, is observed privately. If a policyholder suffers a loss, he chooses to file a claim of some size $\hat{\Theta}(\theta)$ during the period $\left(t_{0}, t_{1}\right)$. In case of honest behavior, the amount of the claim will equal the actual loss, i.e., $\hat{\theta}=\theta$. If the individual decides to defraud, he reports some finite $\hat{\theta}>\theta$. Equation (1) summarizes all values the policyholder can report to the insurance company in the claiming scheme $\hat{\Theta}$ :

$$
\hat{\theta}=\hat{\Theta}(\theta) \begin{cases}=\theta, & \text { no fraud } \\ >\theta, & \text { fraud }\end{cases}
$$


In the course of this paper, we will use the notation

$$
\mathcal{F}=\{\hat{\theta} \mid \hat{\theta}>\theta\}
$$

to denote the set consisting of all fraudulent claims filed by policyholders. The elements in $\mathcal{F}$ are characterized by the individuals' defrauding strategies. We will analyze different scenarios of fraud behavior in the course of this paper.

Insurance companies don't have full information about the exact value of the individual true loss amount. However they can verify claims with auditing. In this paper we assume audit costs $k$ in each claim verification and all auditing processes are perfect, i.e., all fraud can be detected. Furthermore, we assume that as a consequence of detected fraud, the insurance company will reject any indemnification. This procedure is based on the fact that insurers are in many cases contractually released from the obligation to pay indemnities in case of fraudulent behavior. In practice, gradations with regard to the indemnification are possible, i.e., the insurer might decide to pay the full loss amount or parts of it, that is, the loss amount minus some administration cost or penalty. In Section 5, we provide a discussion and additional results in cases where insurance companies do not completely deny the losses. Figure 1 illustrates the interaction of the different processes introduced in this model framework.

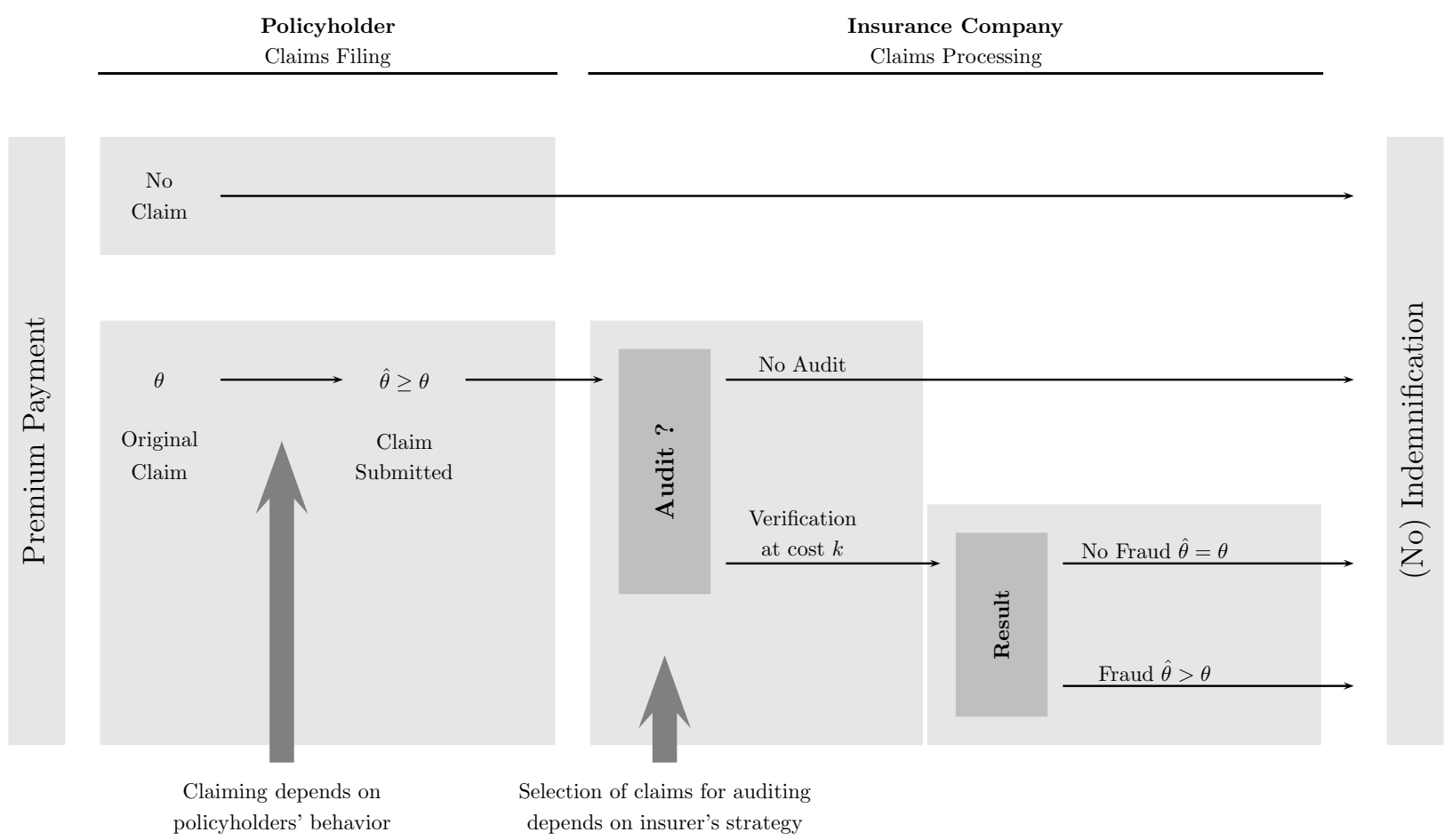

Figure 1: Overview of the processes associated with the filing and handling of insurance claims over the course of one period. An indemnification may or may not be paid out by the end of the period depending on the previous events. 
Following Figure 1, the payment of an indemnification depends on several aspects. On the one hand, the policyholder needs to have suffered an insured loss ${ }^{1}$ during the course of the observation period. Otherwise, he would not have a reason to file a claim. In the case when the claimant decides to defraud, his indemnification is dependent on whether auditing takes place or not: If the reported loss is not verified, he receives the payment $\hat{\theta}$. If the filed claim undergoes an auditing process, however, the attempt to defraud will be revealed and any indemnification payment will be rejected, i.e., both excess and actual loss amount are denied (see the above discussion). If the policyholder belongs to the group of honest individuals, his loss $\theta$ will be indemnified no matter whether auditing took place or not.

Two key elements in the model framework which we have not elaborated on so far are the behavioral strategies of both stakeholders: The amount of the reported claim in case of dishonesty is defined by policyholders' defrauding behavior, while the decision whether or not to initiate a verification process is dependent on the insurance company's auditing strategy. We present and analyze different examples for the respective strategies as well as behavioral adaptations in Sections 3 and 4.

\subsection{Insurance Company: Contribution Margin and Participation Constraint}

From the insurance company's perspective, we observe the future cash flows at the beginning of a period in $t=t_{0}$ and at the time of loss realization and settling at the end of that period in $t=t_{1}$ and analyze the resulting contribution margin per contract $C M$ in $\left(t_{0}, t_{1}\right)$. The insurance company receives a premium payment $P$ from each policyholder in $t=t_{0}$. As already presented in the beginning of Section 2 , the outflows of each period in $t=t_{1}$ depend on whether claims have been filed by the policyholders, and if so, whether they were audited or not and the result of the eventual verification process (see Figure 1).

A key element in this model framework is the insurance company's auditing strategy. It indicates which of the incoming claims are subject to verification and which of them are indemnified without verification. We use the following general notation to denote the auditing strategy

$$
\mathcal{A}=\{\hat{\theta} \mid \hat{\theta} \text { is audited }\}
$$

We distinguish four scenarios which lead to different values in the contribution margin for a single contract $C M(P, k, \hat{\theta}, \mathcal{A}, \mathcal{F})$. Using the notations introduced in Equations (2) and (3), we define

$$
C M(P, k, \hat{\theta}, \mathcal{A}, \mathcal{F})=\left\{\begin{array}{ll}
P & , \text { no loss occurred, i.e., } \hat{\theta}=0 \\
P-\hat{\theta} & , \text { no auditing, i.e., } \hat{\theta} \in \mathcal{A}^{c} \\
P-\hat{\theta}-k & , \text { auditing, but no fraud, i.e., } \hat{\theta} \in \mathcal{A} \cap \mathcal{F}^{c} \\
P-k & , \text { auditing, fraud detected, i.e., } \hat{\theta} \in \mathcal{A} \cap \mathcal{F}
\end{array},\right.
$$

\footnotetext{
${ }^{1}$ Remember that we are considering only situations where fraud is committed through claim buildup and not through planned fraud.
} 
which can also be the stated as:

$$
\begin{aligned}
C M(P, k, \hat{\theta}, \mathcal{A}, \mathcal{F}) & =P-\hat{\theta} \cdot \mathbb{1}_{\mathcal{A}^{c}}(\hat{\theta})-\hat{\theta} \cdot \mathbb{1}_{\mathcal{A} \cap \mathcal{F}^{c}}(\hat{\theta})-k \cdot \mathbb{1}_{\mathcal{A}}(\hat{\theta}) \\
& =P-\hat{\theta}\left[1-\mathbb{1}_{\mathcal{A} \cap \mathcal{F}}(\hat{\theta})\right]-k \cdot \mathbb{1}_{\mathcal{A}}(\hat{\theta})
\end{aligned}
$$

where $\mathbb{1}_{X}(y)$ denotes the indicator function, i.e., it takes the value 1 if $y$ is in the set $X$ or 0 if $y$ is not in $X$.

Hence, in this context $\mathbb{1}_{\mathcal{A}}(\hat{\theta})$ represents the number of claims which were subject to verification whereas $\mathbb{1}_{\mathcal{A}^{c}}(\hat{\theta})$ states the number of claims which were indemnified without auditing. $\mathbb{1}_{\mathcal{A} \cap \mathcal{F}}(\hat{\theta})$ counts the number of cases when fraudulent claims underwent an auditing process, i.e., the number of cases when the attempt to defraud was uncovered while $\mathbb{1}_{\mathcal{A} \cap \mathcal{F}^{c}}(\hat{\theta})$ shows the number of cases when honest claims were verified.

Overall, the insurance company is interested in its entire policyholder population and thus considers the average contribution margin per contract $\mathbb{E}(C M)$, i.e., the net present value of future incoming and outgoing cash flows $N P V$. We define:

$$
N P V=N P V(P, k, \hat{\theta}, \mathcal{A}, \mathcal{F})=\mathbb{E}(C M(P, k, \hat{\theta}, \mathcal{A}, \mathcal{F})),
$$

where $\mathbb{E}(Y)$ denotes the expected value of the stochastic variable $Y$. Hence, we analyze the insurance company's net present value as the expected value of future cash flows discounted at the risk-free rate $r_{f}=0$. This assumption $r_{f}=0$ holds throughout the paper.

Based on Equation (6), we can formulate the insurance company's participation constraint.

Condition 1 The insurance company is willing to maintain the insurance relationship with its policyholder population if the net present value per contract is non-negative:

$$
N P V \geq 0 \text {. }
$$

\subsection{Policyholder: Expected Utility and Participation Constraint}

From the policyholder perspective, we analyze their wealth positions and the corresponding average expected utility at the end of the period $t=t_{1}$ in the framework introduced above. We assume each individual initially to hold the same wealth position $W^{2}$ and to take out the same insurance contract. At the beginning of the observation period in $t=t_{0}$, the payment of the insurance premium $P$ is due. Consequently, each policyholder is endowed with the wealth position $W_{0}^{I}=W-P$, where the superscript $I$ indicates the existence of an insurance contract.

The successive development of this wealth position $W_{0}^{I}$ depends on whether the policyholders suffer an insured loss and, if so, whether they choose to report their loss truthfully or not and whether their

\footnotetext{
${ }^{2}$ Since the actual value of the initial wealth position will have no impact on the decision whether to maintain the insurance relationship or not, the policyholders might even be endowed with different wealth positions. We assume the latter to be invested safely with the risk-free rate $r_{f}=0$.
} 
fraud is revealed in case of dishonesty or not. Taking the occurrence of loss into account, the wealth position at the end of the observation period in $t=t_{1}$ is given by

$$
W_{1}^{I}=W-P-\theta+\hat{\theta}\left[1-\mathbb{1}_{\mathcal{A} \cap \mathcal{F}}(\hat{\theta})\right]
$$

where $W$ is invested without risk with $r_{f}=0$. If no insured loss has occurred throughout the observation period, Equation (8) simplifies to $W_{1}^{I}=W_{0}^{I}=W-P$.

In order to be able to formulate a participation constraint from the policyholder perspective, we consider the development of their wealth position throughout the observation period if they have not signed an insurance contract prior to the occurrence of loss. In $t=t_{0}$, the individuals would not have to make a payment in the amount of the insurance premium. Hence, the corresponding wealth position is given by $W_{0}^{N}=W$, where the superscript $N$ implies the absence of an insurance relationship. If some $\operatorname{loss} \theta$ occurs by $t=t_{1}$, this amount decreases to $W_{1}^{N}=W_{0}^{N}-\theta=W-\theta$, whereas, without loss during the observation period, we have $W_{1}^{N}=W_{0}^{N}=W$.

We assume policyholders' expected utility to be described by a standard mean-variance utility function of the corresponding wealth position. The degree of risk aversion is expressed by the parameter $a(>0)$. Generally, for a given stochastic wealth position $Z$, its expected utility for the individual is given by $U(Z)=\mathbb{E}(Z)-\frac{a}{2} \operatorname{Var}(Z)$, where $\operatorname{Var}(Z)$ denotes the variance of the stochastic variable $Z$.

Using the equations above and considering that the probability of loss occurrence is denoted by $\pi$, the final expected utility if there is no insurance contract can be written as

$$
U\left(W_{1}^{N}\right)=W-\pi \mathbb{E}(\theta)-\frac{a}{2} \pi^{2} \operatorname{Var}(\theta)
$$

Similarly, for the setting where there is already an insurance relationship between policyholders and insurance company, the final expected utility is given by

$$
U\left(W_{1}^{I}\right)=W-P-\mathbb{E}\left(\pi \theta-\hat{\theta}\left[1-\mathbb{1}_{\mathcal{A} \cap \mathcal{F}}(\hat{\theta})\right]\right)-\frac{a}{2} \operatorname{Var}\left(\pi \theta-\hat{\theta}\left[1-\mathbb{1}_{\mathcal{A} \cap \mathcal{F}}(\hat{\theta})\right]\right) .
$$

Comparing Equation (10) with Equation (9) results in the policyholders' participation constraint. For this purpose, we introduce the notion of the gain in expected utility from having signed an insurance contract

$$
\Delta U=U\left(W_{1}^{I}\right)-U\left(W_{1}^{N}\right)
$$

Condition 2 Policyholders are willing to maintain the insurance relationship with the insurance company if their final expected utility is greater as a result of having insurance than without it, i.e.,

$$
\Delta U \geq 0
$$

We want to point out that policyholders' participation constraint functions the same in the case of a multi-period model, i.e., they do not cancel the insurance relationship unless their gain in expected utility from having insurance coverage $\Delta U$ is negative. In particular, the insurance company is in a position to optimize its position while policyholders would choose to cancel their insurance contracts only if $\Delta U<0$. 


\subsection{Optimization Problem}

Summing up the information we have presented so far with regard to the model framework as well as the insurance company's and the policyholders' participation constraints, we can formulate the resulting optimization problem.

The insurance company is aiming to derive an auditing strategy $\mathcal{A}$ such that its net present value of future cash flows $N P V$ is maximized. At the same time, it needs to be ensured that the stakeholders are willing to maintain their insurance relationships, i.e., Equations (7) and (12) hold. Formally, this objective is given by

$$
\left\{\begin{array}{l}
\max _{\mathcal{A}} N P V(P, k, \hat{\theta}, \mathcal{F}, \mathcal{A}) \\
N P V \geq 0 \\
\Delta U \geq 0
\end{array} .\right.
$$

We make the assumption that the former charges the given market premium $P$. In particular, this implies that an adaptation of the insurance premium as part of the fraud handling is not feasible. Furthermore, we assume no interaction with the number of acquired contracts.

\section{Optimal Auditing Strategies}

Section 2 constitutes the introduction of the model framework as well as the insurance company's and policyholders' behavioral strategies in general. On that basis, we first introduce the basic policyholder claiming (and defrauding) scheme in Section 3.1 and the insurance company auditing strategy in Section 3.2. Both schemes make use of several parameters and thresholds defining the behavior in our model. Using both models for policyholder and insurance company we consider the interaction between the insurer and policyholders and define their adaptation over multiple periods in Section 3.3. In Section 3.4 we present the numerical implementation of the iterative adaptation and optimization in four steps.

\subsection{Policyholder Claiming Scheme}

As discussed above, we consider insurance fraud in the form of buildup, i.e., if there is a loss, some policyholders will decide to file a fraudulent exaggerated claim if this appears profitable.

In the context of our study, we assume the fraud amount from the inflation of the magnitude of loss is not a decision variable for policyholders. In case an individual decides to engage in fraudulent activities, he or she will consider the buildup as a percentage 'surcharge' on the actual loss amount instead of filing some value which deviates significantly from the actual loss amount. This approach increases the likelihood of a fraudulent claim being perceived as legitimate by the insurance company, and thus avoiding an audit. This assumption is in line with Viaene and Dedene (2004) who find that policyholders involved in soft fraud typically tend to file claims containing small fraud amounts. Further evidence can be found in the behavioral economic theory literature. Individuals weigh the consequences accompanied by losses more heavily than the ones from a gain of the same size (see, e.g., Kahneman and Tversky (1979), Kerr (2012)). Applied to the context of our model framework this implies that the loss from being caught 
committing soft fraud (i.e., indemnification is waived completely) is perceived as a higher burden than the potential profit from a successful buildup attempt. Another topic that is discussed in the literature (not included in our model) concerns buildup, as a surcharge on the loss suffered gives policyholders the opportunity to 'back down and claim the appropriate amount' if investigated by the insurance company rather than in the case of outright fraud (see, e.g., Emerson (1992)).

From the perspective of repair shops, which might also be involved in fraudulent activities, extensive inflations of the loss amount seem to be unlikely as well. According to Hubbard (2002), service providers in general have a reputational incentive to act in their clients' favor since the latter tend to return more often if they are satisfied with previous services. These findings are in line with statements made by experts from an insurance company whose experiences have shown that repair shops focus on the actual loss amount when trying to charge too much for certain services. This way even exaggerated claims seem legitimate and are less likely to undergo an auditing process resulting in direct indemnification.

As part of the defrauding strategy, we assume that whenever policyholders have suffered a loss during the observation period they report back a multiple of the actual loss amount. We denote this constant multiplicative factor by $\alpha=\hat{\theta} / \theta$. However, it is well known that insurance companies perform audits in order to verify the truthfulness of incoming claims. Consequently, fraud-prone policyholders can be expected to adjust their fraud strategy accordingly. We assume that all of these individuals have an inner threshold value for defrauding $\hat{\theta}_{\mathrm{ph}}^{*}$. With regard to their fraud strategy this threshold value implies that they apply their fraud strategy $\alpha$ up to the preset threshold value $\hat{\theta}_{\mathrm{ph}}^{*}$. To be more precise, if the amount of the actual loss $\theta$ is smaller than the threshold $\hat{\theta}_{\mathrm{ph}}^{*}$, the minimum of $\alpha \theta$ and $\hat{\theta}_{\mathrm{ph}}^{*}$ is reported to the insurance company. However, if the amount of the loss suffered already exceeds that threshold, the policyholder claims this amount truthfully. We can write this strategy as

$$
\hat{\theta}=\hat{\Theta}\left(\theta, \alpha, \hat{\theta}_{\mathrm{ph}}^{*}\right)= \begin{cases}0 & , \text { no loss occurred } \\ \theta & , \text { no fraud or } \theta>\hat{\theta}_{\mathrm{ph}}^{*} \\ \min \left\{\alpha \theta, \hat{\theta}_{\mathrm{ph}}^{*}\right\} & , \text { fraud and } \theta<\hat{\theta}_{\mathrm{ph}}^{*}\end{cases}
$$

The set consisting of all fraudulent claims associated with this strategy $\hat{\Theta}\left(\theta, \alpha, \hat{\theta}_{\mathrm{ph}}^{*}\right)$ is denoted by $\mathcal{F}_{\alpha, \hat{\theta}_{\mathrm{ph}}^{*}}$.

The question arises as to how policyholders determine an adequate threshold value for defrauding. Here we refer to the data presented in Section 1 regarding survey reports indicating that policyholders may use independent contractors, such as repair shops, to act as their partners in fraud. These repair shops are likely to have developed insight about the value of $\hat{\theta}^{*}$ due to repeated experiences in repairing cars for insured damages. Based on this information they can make proper assumptions concerning the insurance companies' prevalent auditing strategies. There are two potential ways repair shops can proceed: provide too much service or bill too much for a particular service (see Dulleck and Kerschbamer (2006)). A particular phenomenon is to include the cost to fix existing damages when repairing the ones which were caused by a current insured event. Such actions might often be undertaken even without the knowledge of the policyholder. On the one hand, the latter might not have the incentive to carefully check the work performed by repair shops since the bill is passed on to the insurance company (see 
Tennyson (2008)). On the other hand, the policyholders might lack the necessary know-how to do so (see Dulleck and Kerschbamer (2006)). Policyholders, however, particularly those who are willing to defraud if it appears to be advantageous, may also seek information themselves to adapt their claiming behavior for purposes of defrauding the insurer. They may seek out others for ideas of successful fraud or perhaps be approached by third parties to participate in defrauding activities. Staying in the field of auto insurance, fraudulent activities may be performed by mutual agreement between the policyholder and the corresponding repair shop, who then share the excess insured payments. In particular, it is possible to adjust policyholders' defrauding strategy as presented in Equation (14). In this respect, we make two important assumptions in our model setup: Firstly, it is assumed that neither the policyholders nor a third party other than the insurance company itself hold exact information concerning the prevalent auditing strategy. Otherwise, the audit would be redundant. Secondly, if a third party (e.g. a repair shop) is involved, the third party always acts in favor of the policyholder and in accordance with the defrauding strategy described in Equation (14). Cases in which the defrauding activity is carried out by the third party for their own purposes are ruled out in the modeling framework, even though such incidents as described above are relevant in insurance practice too.

\subsection{Insurance Company Auditing Strategy}

From the insurance company perspective, this fraud strategy implies the need to adjust its auditing strategy $\mathcal{A}$. After having performed audits for at least one period, the company should have gained enough information on policyholder fraud behavior to do so. Revealed fraud can serve as an especially helpful information base for improving the existing auditing strategy. Based on an insurance company's partial knowledge about many claims and payment ranges for claims, that is, assuming that a sufficiently high number of verification processes has been performed (which has revealed sufficiently numerous defrauding attempts), the insurer will note that fraudulent claims do not exceed a certain threshold value. Hence, it is unnecessary to audit claims above that value, allowing insurers instead to verify medium-sized claims. Consequently, in this case the aim is to result in the optimal auditing range $\mathcal{A}_{\mathrm{R}}$. The upper bound of this range would have to be the policyholders' persistent inner threshold value $\hat{\theta}_{\mathrm{ph}}^{*}$. Unfortunately, the insurance company does not have this information. However, an adequate estimate for this value is the maximum fraud amount which was detected during the last period. We denote this value by $\hat{\theta}_{\max }$ and formally define it as

$$
\hat{\theta}_{\max }=\max \left\{\hat{\theta} \cdot \mathbb{1}_{\mathcal{A} \cap \mathcal{F}}\right\} .
$$

Setting the upper bound of the auditing range $\mathcal{A}_{\mathrm{R}}$ to $\hat{\theta}_{\max }$ practically implies that no incoming claim above that value will be verified. However, since one cannot be absolutely sure whether the actual maximum of all fraudulent claims has been determined, i.e., whether or not fraud is being committed beyond $\hat{\theta}_{\max }$, it is reasonable to include a safety margin $s>0$. Consequently, the adjusted upper bound of the auditing range is set $(1+s) \cdot \hat{\theta}_{\max }$. 
The second parameter that characterizes the audit range $\mathcal{A}_{\mathrm{R}}$ is its lower bound, which is expressed by $\hat{\theta}_{\mathrm{R}}^{*}$. Summing up, we can formulate this auditing strategy as

$$
\mathcal{A}_{\mathrm{R}}=\mathcal{A}_{\mathrm{R}}\left(\hat{\theta}_{\mathrm{R}}^{*}, s, \hat{\theta}_{\max }\right)=\left\{\hat{\theta} \mid \hat{\theta}_{\mathrm{R}}^{*} \leq \hat{\theta} \leq(1+s) \cdot \hat{\theta}_{\max }\right\}
$$

We can specify the optimization problem given in Equation (13) with regard to this new setting. The aim here is to derive the optimal lower bound $\hat{\theta}_{\mathrm{R}}^{*}$ of the auditing range such that the insurance company's net present value $N P V$ is maximized:

$$
\left\{\begin{array}{l}
\max _{\hat{\theta}_{\mathrm{R}}^{*}} N P V\left(P, k, \hat{\theta}, \mathcal{F}_{\alpha, \hat{\theta}_{\mathrm{ph}}^{*}}, \mathcal{A}_{\mathrm{R}}\right) \\
N P V \geq 0 \\
\Delta U \geq 0 .
\end{array}\right.
$$

As already mentioned above, the auditing strategy $\mathcal{A}_{\mathrm{R}}$ requires the availability of information with regard to the prevalent defrauding behavior, i.e., $\hat{\theta}_{\text {max }}$ needs to be determined. Consequently, $\mathcal{A}_{\mathrm{R}}$ is applicable after the first observation period at the earliest when enough audits have been performed to specify the value of $\hat{\theta}_{\max }$. However, this implies the need for a different verification scheme for the very first observation period. In our case, we assume that the insurance company will revert to an initial strategy for auditing during the first period. This one is characterized by a threshold value $\hat{\theta}_{\text {init }}^{*}$, i.e., all incoming claims which exceed this threshold are subject to verification whereas claims whose amount is below this indicator will be indemnified right away. We denote this specific auditing strategy by $\mathcal{A}_{\text {init }}$ and define it formally as

$$
\mathcal{A}_{\text {init }}=\mathcal{A}_{\text {init }}\left(\hat{\theta}_{\text {init }}^{*}\right)=\left\{\hat{\theta} \mid \hat{\theta}_{\text {init }}^{*} \leq \hat{\theta}\right\}
$$

Then, after enough information has been gathered during the first observation period, the insurer switches to the auditing range $\mathcal{A}_{\mathrm{R}}$ in the subsequent periods.

\subsection{Behavioral Adaptation}

So far, the policyholders in the population who are likely and willing to defraud were assumed to adhere to a constant fraud strategy. They chose a constant multiplicative factor $\alpha$ and/or the same threshold value for defrauding $\hat{\theta}_{\mathrm{ph}}^{*}$ over the course of several periods. This constancy results from the assumption that the policyholder population does not obtain any information on the insurance company's prevalent verification process. Hence, there was no need for an adjustment of their behavior. That also had an impact on the insurance company's corresponding optimal auditing strategy. As soon as the optimal verification process with respect to the prevalent defrauding behavior was found, i.e., right after the first observation period, there was no need for the insurance company to perform any adjustments to it. In summary, none of the participants in the insurance relationship had to change their behavior.

However, it is realistic to assume that there might be signals indicating whether the insurance company has changed its auditing strategy in subsequent periods or not. Especially in the case of automobile insurance, signaling is issued by (authorized) repair shops. Because they deal with a large number of 
insured events, they are able to estimate changes in the auditing behavior of different insurance companies. In these cases, policyholders who are prone to defrauding would be given a chance to change their fraud behavior and react to the new verification scheme. If the insurance company announces strengthened controls in order to combat insurance claims fraud, the fraudulent part of the policyholder population would choose to act more carefully in terms of their defrauding strategy, i.e., lower the multiplicative factor $\alpha$ and/or their threshold value for defrauding $\hat{\theta}_{\mathrm{ph}}^{*}$. In the opposite case, knowing that the insurance company will relax their auditing scheme, it can be assumed that attempts are made to exaggerate the actual loss amount $\theta$ even more and obtain higher indemnification payments, i.e., by increasing $\alpha$ and/or $\hat{\theta}_{\mathrm{ph}}^{*}$.

Figure 2 gives an overview of the interaction between insurance company and (defrauding) policyholders and the resulting adjustment processes of the respective behavioral strategies.

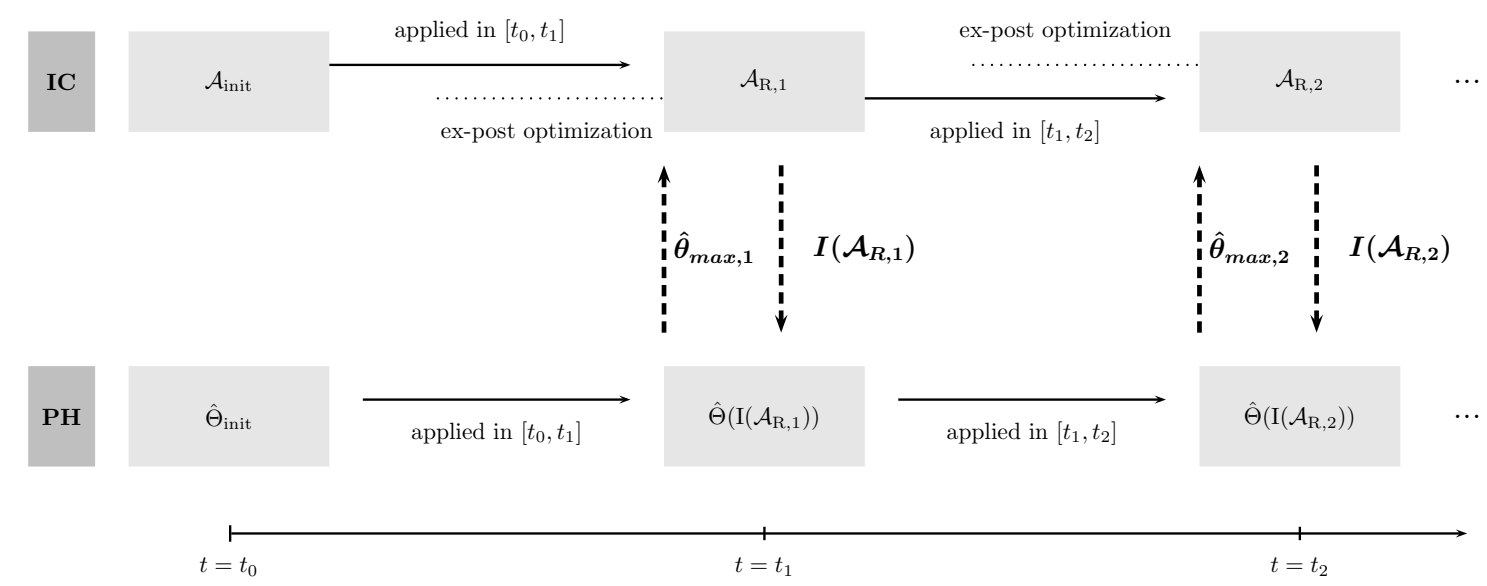

Figure 2: Interaction between insurance company (IC) and policyholders (PH) over the course of the first two periods in an insurance relationship.

Both stakeholders define their respective initial strategy at the beginning of the very first observation period in $t=t_{0}$. For the insurance company, this is its initial auditing strategy denoted by $\mathcal{A}_{\text {init }}$. Since by that point in time, no information regarding the policyholders' defrauding strategy is available, it will be characterized by an initial threshold value for auditing $\hat{\theta}_{\text {init }}^{*}$. The policyholders themselves choose a claiming scheme $\hat{\Theta}_{\text {init. }}{ }^{3}$ In particular, the initial fraud strategy is defined by the multiplicative factor $\alpha$ and an initial threshold value for defrauding $\hat{\theta}_{\mathrm{ph}, 0}^{*}$ (see Equation (14)). These two strategies are applied throughout the first observation period $\left[t_{0}, t_{1}\right]$. At its end, in $t=t_{1}$, all information with regard to the actual distribution of the claimed losses throughout that period as well as indications on policyholders' defrauding scheme, i.e., the maximum value of detected fraud $\hat{\theta}_{\max , 1}$ in $\left[t_{0}, t_{1}\right]$, are available to the insurance company. ${ }^{4}$ Based on this information, the insurer determines its optimal auditing strategy

\footnotetext{
${ }^{3}$ The function $\hat{\Theta}$ includes the defrauding strategy for dishonest policyholders (see Equation (14)).

${ }^{4}$ We assume that a sufficiently high number of audits has been performed during the course of the first period which has resulted in the detection of fraudulent claims, i.e., $\hat{\theta}_{\max , 1}$ is observed.
} 
$\mathcal{A}_{\mathrm{R}, 1}$ for the first observation period ex-post. The corresponding optimization problem is defined in Equation (17). This adjusted auditing scheme will then be applied in the second observation period $\left[t_{1}, t_{2}\right]$. At the same time, some signal $I\left(\mathcal{A}_{\mathrm{R}, 1}\right)$ concerning the adjustment of the verification process is communicated to the policyholder population. They themselves now have the opportunity to adapt their behavior accordingly. While honest policyholders adhere to reporting the actual loss amount when they have suffered an insured loss, the ones who are willing to defraud adjust their threshold value for defrauding to $\hat{\theta}_{\mathrm{ph}, 1}^{*}$. This new claiming scheme will be denoted by $\hat{\Theta}\left(I\left(\mathcal{A}_{\mathrm{R}, 1}\right)\right)$ and is applied in the second observation period $\left[t_{1}, t_{2}\right]$. However, the change in defrauding behavior will be registered by the insurance company in the form of a different maximum value of detected fraud $\hat{\theta}_{\max , 2}$ in the course of that second period. This new piece of information on policyholders' behavior results once again in an ex-post optimization of the prevalent auditing strategy to $\mathcal{A}_{\mathrm{R}, 2}$ at the end of this very period in $t=t_{2}$. Again, the adjusted verification scheme $\mathcal{A}_{\mathrm{R}, 2}$ is applied in the following observation period after the policyholder population has been provided with a signal $I\left(\mathcal{A}_{\mathrm{R}, 2}\right)$ concerning the change in auditing. The interaction and adaptation processes can be repeated in the same fashion over the course of several periods.

It needs to be emphasized that while we focus on the derivation of the optimal auditing strategies $\mathcal{A}_{\mathrm{R}, n}$, we also assure that all participants are willing to maintain the insurance relationship, i.e., all participation constraints as defined in Equations (7) and (12) need to hold when applying the optimal verification process.

\subsection{Numerical Implementation of Iterative Optimization}

In this subsection, we present the iterative approach regarding the optimization of the auditing range $\mathcal{A}_{\mathrm{R}, n}$ in the $n^{\text {th }}$ period from the insurance company perspective when interaction and hence adaptation are observable. ${ }^{5}$ Even without the behavioral adaptation presented in Section 3.3, a numerical optimization procedure is necessary since an analytical solution for the optimization problem formalized in Equation (17) cannot be found. This is due to the fact that a general functional relationship for the indicator variable introduced in Equation (5) and used in the target function and the restrictions of the optimization problem (17) cannot be obtained without further limiting assumptions. More precisely, the probabilities needed for the indicator variable are a function of the relative fraud amount $\alpha$.

Step 1: Adjustment of claiming scheme We first consider the policyholders' claiming scheme $\hat{\Theta}_{n}\left(\theta_{n}, \alpha, \hat{\theta}_{\mathrm{ph}, n}^{*}\right)$ is applied at the beginning of each iteration. It is characterized by the defrauders' strategy indicated by a constant multiplicative factor $\alpha$ and a threshold value for defrauding $\hat{\theta}_{\mathrm{ph}, n}^{*}$ which is adjusted

\footnotetext{
${ }^{5}$ The subscripts $n$ in the course of this subsection indicate the $n^{\text {th }}$ iteration process, i.e., the respective quantities for the $n^{\text {th }}$ observation period. In doing so, we consider all $n \geq 2$. The special case of $n=1$ corresponds to the initial period.
} 
each period based on the signal $I\left(\mathcal{A}_{\mathrm{R}, n-1}\right)$. In accordance with Equation (14), for the policyholders' claiming scheme in the $n^{\text {th }}$ period we obtain

$$
\hat{\theta}_{n}=\hat{\Theta}_{n}\left(\theta_{n}, \alpha, \hat{\theta}_{\mathrm{ph}, n}^{*}\right)= \begin{cases}0 & , \text { no loss occurred } \\ \theta_{n} & , \text { no fraud or } \theta_{n}>\hat{\theta}_{\mathrm{ph}, n}^{*}, \\ \min \left\{\alpha \theta_{n}, \hat{\theta}_{\mathrm{ph}, n}^{*}\right\} & , \text { fraud and } \theta_{n}<\hat{\theta}_{\mathrm{ph}, n}^{*}\end{cases}
$$

where $\theta_{n}$ represents a realization of the loss variable in the $n^{\text {th }}$ period. The set consisting of all fraudulent claims associated with this strategy is denoted by $\mathcal{F}_{\alpha, \hat{\theta}_{\mathrm{ph}, n}^{*}}$.

Step 2: Determining maximum value of detected fraud The identification of the maximum fraud value which was actually detected in the $n^{\text {th }}$ iteration, i.e., period, is the focus of this step. Apparently, it depends on the auditing strategy $\mathcal{A}_{\mathrm{R}, n-1}$ which was derived to be optimal in the $(n-1)^{\text {th }}$ period and is then applied in the $n^{\text {th }}$ period. Using the notation presented in Equation (5), the maximum value of detected fraud in the $n^{\text {th }}$ period can be defined as

$$
\hat{\theta}_{\max , n}=\max \left\{\hat{\theta}_{n} \cdot \mathbb{1}_{\mathcal{A}_{\mathrm{R}, n-1} \cap \mathcal{F}_{\alpha, \hat{\theta}_{\mathrm{ph}, n}^{*}}}\left(\hat{\theta}_{n}\right)\right\} .
$$

Step 3: Ex-post optimization of auditing strategy The value of $\hat{\theta}_{\max , n}$ forms the basis for the actual optimization process. Considering Equation (16), the insurance company's auditing strategy for the $n^{\text {th }}$ period is given by

$$
\mathcal{A}_{\mathrm{R}, n}=\left\{\hat{\theta}_{n} \mid \hat{\theta}_{\mathrm{R}, n}^{*} \leq \hat{\theta}_{n} \leq(1+s) \cdot \hat{\theta}_{\mathrm{max}, n}\right\},
$$

with $s$ being the safety margin.

The aim is now to find the lower bound $\hat{\theta}_{\mathrm{R}, n}^{*}$ of this audit range such that the insurance company's net present value of future cash flows $N P V$ is maximized and the stakeholders' participation constraints hold:

$$
\left\{\begin{array}{l}
\max _{\hat{\theta}_{\mathrm{R}, n}^{*}} N P V\left(P, k, \hat{\theta}, \mathcal{F}_{\alpha, \hat{\theta}_{\mathrm{ph}, n}^{*}}, \mathcal{A}_{\mathrm{R}, n}\right) \\
N P V_{n} \geq 0 \\
\Delta U_{n} \geq 0
\end{array}\right.
$$

where $\Delta U_{n}$ denotes the policyholders' gain in expected utility from having insurance coverage in the $n^{\text {th }}$ period.

Step 4: Communication of signal After having found the optimum value for the lower bound $\hat{\theta}_{\mathrm{R}, n}^{*}$ of the auditing range, i.e., the optimal auditing strategy $\mathcal{A}_{\mathrm{R}, n}$, at the end of observation period $n$, a 
signal $I\left(\mathcal{A}_{\mathrm{R}, n}\right)$ is communicated to the policyholder population informing them of the adjustment of the prevalent verification scheme. For this purpose, we define this signal as follows:

$$
I\left(\mathcal{A}_{\mathrm{R}, n}\right)=\left(\frac{\hat{\theta}_{\mathrm{R}, n}^{*}}{\hat{\theta}_{\mathrm{R}, n-1}^{*}}+\frac{\hat{\theta}_{\mathrm{max}, n}}{\hat{\theta}_{\max , n-1}}\right) / 2 .
$$

This signal is used to adapt the claiming scheme $\hat{\Theta}_{n+1}$ at the beginning of period $n+1$. In this context, the signal $I$ is an average of two ratios: The first one represents the change of the upper bound from period $n-1$ to $n$ whereas the second one constitutes the change of the maximum value of detected fraud from period $n-1$ to $n$.

\section{Simulation Results}

In Sections 2 and 3, we presented a model framework and potential optimal auditing strategies when interaction between policyholder and insurance company and consequently behavioral adaptation over the course of several observation periods is possible. In this section, we present and analyze the corresponding numerical solutions for the optimal auditing strategy from the insurance company perspective. We first describe the parameterization used for our reference setting in Section 4.1. Then, we provide numerical results and sensitivity analyses in Section 4.2. We derive information on the optimal auditing range $\mathcal{A}_{\mathrm{R}}$, the number of performed audits, the number of fraudulent claims and the insurer's net present value $N P V$. Sensitivity analyses focus on the variation in the cost per audit $k$, the relative fraud amount $\alpha$ and the policyholders initial threshold value $\hat{\theta}_{\mathrm{ph}}^{*}$. In all cases, the optimal auditing range $\mathcal{A}_{\mathrm{R}}$, the policyholders utility gain $\Delta U$ and the insurer's net present value $N P V$ are reported.

\subsection{Parametrization of the Reference Setting}

In our simulations, we consider a policyholder population consisting of $M=2500000$ individuals. An assumed probability of loss occurrence of $\pi=0.2$ leads to $N=500000$ loss realizations of $\theta$ per observation period. In doing so, the losses are sampled from a log-normal distribution. This assumption is commonly used as mentioned in Marlin (1984) since it guarantees positive values for the realization of the random variable. In particular, the expected value $\mathbb{E}(\theta)$ is set to $\mu=1$ and the variance $\operatorname{Var}(\theta)=\sigma^{2}=0.4$. All individuals among the population are assumed to be risk averse. For instance, their risk aversion parameter $a$ is considered to be 6 . Furthermore, the policyholders' initial wealth position is set to $W=0$. At the same time, the individuals have to pay an insurance premium $P$ at the beginning of each observation period. The latter can be split into the fair premium and an appropriate loading factor. The fair premium corresponds to the expected loss. Hence, having set the expected value of the loss variable $\theta$ to $\mu=1$ and considering the probability of suffering a loss to be $\pi=0.2$, this implies a fair premium of 0.2 . However, since the insurance company faces additional costs, it will add a corresponding loading factor to the fair premium. As mentioned in Cummins and Mahul (2004), the loading factor cannot be too large since potential policyholders would not sign the insurance contract under such conditions. For our analyses, we will assume the total insurance premium to be $P=0.3$. Furthermore, the cost per audit 
is set to $k=0.05$ which corresponds to $16.67 \%$ of the insurance premium $P$. For the purpose of our analyses, we will disregard costs other than the ones due to auditing. The share of fraud-prone policyholders among the population is assigned the value $p=0.2$, i.e., $20 \%$ of all policyholders who suffer an insured loss who may exaggerate that amount if it is in accordance with their defrauding strategy. Their defrauding strategy is accompanied by the choice of the relative fraud amount $\alpha$ and/or an appropriate threshold value for defrauding $\hat{\theta}_{\mathrm{ph}}^{*}$. To start with, we take $\alpha$ to be 2, i.e., the policyholders who decide to defraud report back an amount twice as high as the actual loss amount. However, if the individuals have a threshold value for defrauding as described in Section 3.1, they never claim more than that value $\hat{\theta}_{\mathrm{ph}}^{*}$. For the purpose of our analyses, we assume $\hat{\theta}_{\mathrm{ph}}^{*}=1.1$ which is $10 \%$ higher than the expected value of the loss variable. Finally, the insurance company needs to decide on the parameters for its auditing strategy $\mathcal{A}$. During the very first period, it opts for a verification process $\mathcal{A}_{\text {init }}$ which is characterized by a threshold value for auditing $\hat{\theta}_{\text {init }}^{*}$. We set its initial value to 1 , which corresponds to the expected value of the loss variable $\theta$. As already explained at the end of Section 3.2, information with regard to policyholders' defrauding behavior needs to be gathered first before being able to apply this verification scheme $\mathcal{A}_{\mathrm{R}}$. Hence, we initially set $\hat{\theta}_{\text {init }}^{*}=1$, and determine the paramters $\hat{\theta}_{\mathrm{max}}$ and $\hat{\theta}_{\mathrm{R}}^{*}$ based on the information obtained based on the first program run. With regard to the upper bound of the auditing range, the value for the safety margin $s$ is assumed to be 0.1 , i.e., the upper bound of the auditing range is $10 \%$ higher than maximum of detected fraud $\hat{\theta}_{\text {max }}$. The resulting auditing range $\mathcal{A}_{\mathrm{R}}$ is then applied in a subsequent program run.

Table 1 sums up the choices for the input parameters for the reference setting as introduced above. In the course of this and the following sections, we base our simulations and studies on these values.

\begin{tabular}{lcr}
\hline Input parameter & & Reference level \\
\hline Total number of policyholders & $M$ & 2500000 \\
Number of loss realizations & $N$ & 500000 \\
Loss distribution & $\theta$ & $\ln \mathcal{N}(1,0.4)$ \\
Insurance premium & $P$ & 0.3 \\
Share of fraud-prone policyholders & $p$ & 0.2 \\
Relative fraud amount & $\alpha$ & 2 \\
Initial threshold value for auditing & $\hat{\theta}_{\text {init }}^{*}$ & 1 \\
Safety margin & $s$ & 0.1 \\
Policyholder's initial threshold & $\hat{\theta}_{\mathrm{ph}, 0}^{*}$ & 1.1 \\
Auditing cost & $k$ & 0.05 \\
Risk aversion parameter & $a$ & 6 \\
\hline
\end{tabular}

Table 1: Input parameters for the reference setting. 


\subsection{Simulation Results and Sensitivity Analyses}

The remainder of this section constitutes the presentation and discussion of the simulation results. For the simulations, we adhere to the parameter choice presented in Table 1 unless noted otherwise.

\subsubsection{Development of Optimization Results Over Several Iterations}

In this subsection, we present and discuss the development of the optimal auditing range $\mathcal{A}_{\mathrm{R}}$ over the course of several iterations. Furthermore, we analyze its impact on quantities like the number of performed audits, the amount of fraudulent claims, the net present value $N P V$ and the gain in utility $\Delta U$. To get a better insight into the effects, we consider both the parametrization of the reference setting with costs per audit $k=0.05$ as well as the case when the costs per audit are raised to $k=0.3$ and compare the results.

\section{Development of Optimal Auditing Range $\mathcal{A}_{\mathbf{R}}$}

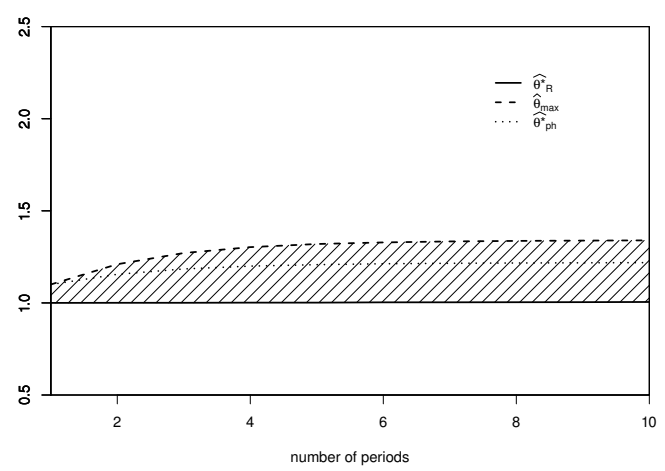

(a) Development of optimal auditing strategy, $k=0.05$

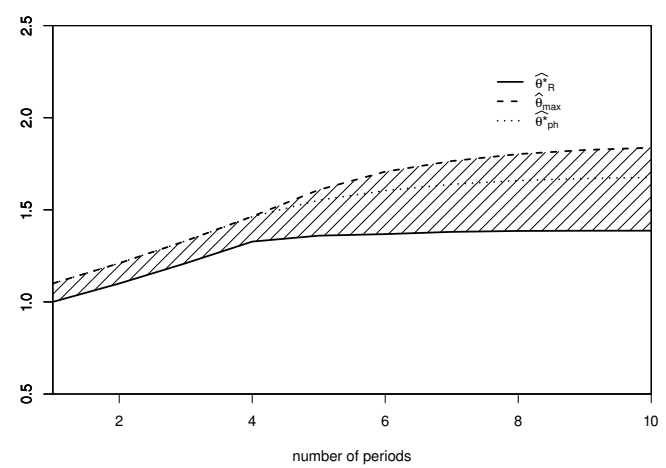

(b) Development of optimal auditing strategy, $k=0.3$

Figure 3: Development of the optimal auditing strategy throughout the course of several iterations for two different choices of the cost per audit $k$. The remaining parameters are chosen as presented in Table 1 .

Comparing Figure 3(a) with Figure 3(b), we can see that higher costs per audit $k$ result in a slightly broader optimal auditing range $\mathcal{A}_{\mathrm{R}}$, i.e., the share of values which may be verified becomes greater. At the same time, the optimal auditing range shifts in an upward direction, i.e., the value of the claims which may be subject to auditing becomes higher. For the insurance company this implies that, in case of high expenditures per case, they should focus their investigations on those claim which exhibit high saving potential whenever an engagement in fraudulent activities is detected.

From the policyholder perspective, we find that higher costs per audit $k$ lead to a higher threshold of defrauding $\hat{\theta}_{\mathrm{ph}}^{*}$, i.e., the value up to which policyholders take buildups into consideration increases. This observation shows that the cost per audit not only has an impact on insurance companies' auditing strategies, but also indirectly on policyholders' behavior, in particular on their defrauding strategy. Since the insurer signals an upward shift in its verification behavior, policyholders (and the corresponding 
service providers) are left with the impression that inflating a loss amount up to some value is more likely to remain undetected than in the previous period. In turn, they then raise their threshold value for defrauding.

\section{Development of Number of Performed Audits and Fraudulent Claims}

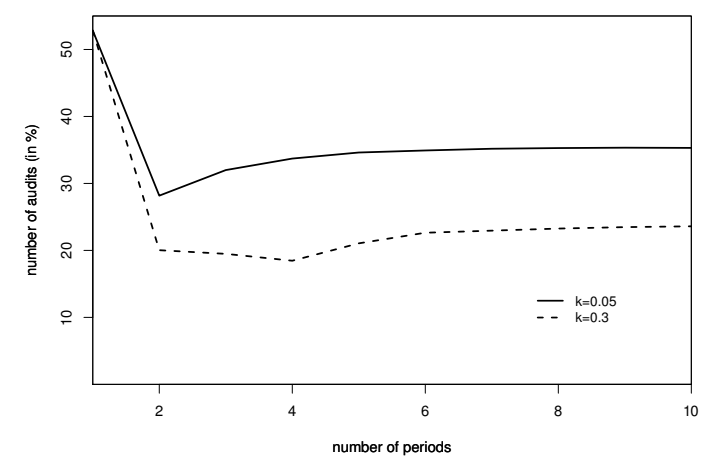

(a) Development of the number of audits performed by the insurance company when applying the optimal auditing strategy for the respective period.

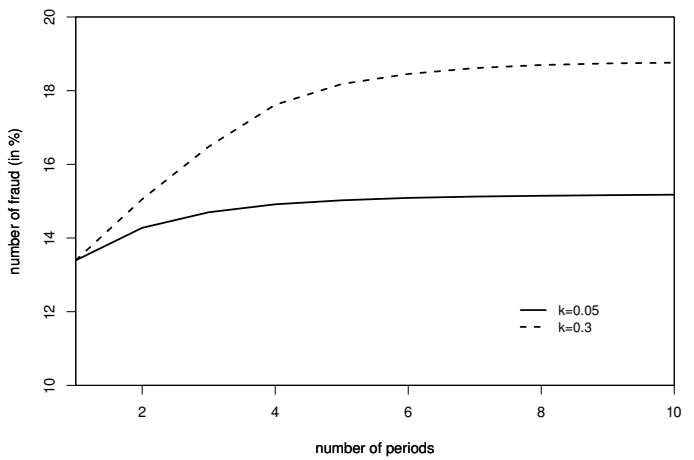

(b) Development of the number of fraudulent claims when the insurance company applies the optimal auditing strategy for the respective period.

Figure 4: Development of number of audits and the number of fraudulent claims over the course of several iterations measured in relation to total number of losses, i.e., filed claims. The development is illustrated for two different choices of the cost per audit $k$. The remaining parameters are chosen as presented in Table 1.

We measure both the number of performed audits and the number of fraudulent claims in relation to the total number of filed claims.

Figure 4(a) confirms the intuition that higher costs per audit $k$ result in a lower share of incoming claims which are subject to verification, i.e., fewer auditing processes are performed. As mentioned above, this restriction in the number of verification processes leads insurance companies to focus on those claims which, in case of detected fraud, have a higher saving potential, i.e., claims with a higher value.

From the policyholder point of view, the number of fraudulent claims consistently increases (see Figure 4(b)). This finding is in line with our results presented in Figure 3. Because they receive a signal indicating an upward shift, the policyholders themselves raise their threshold value for defrauding. As a consequence of this elevation, the number of losses below the threshold increases, resulting in more cases where actions are taken to inflate the claim amount.

It needs to be noted that even though $20 \%$ of the policyholder population is willing to exaggerate its loss amount, the actual number is below that value (see Figure 4(b)). This phenomenon can be explained by the existence of the threshold value for defrauding $\hat{\theta}_{\mathrm{ph}}^{*}$ up to which fraud is actually taken into consideration. The actual share of buildup among all claims may depend on different factors. As already seen in Figure 3, higher costs per audit $k$ result in an increased defrauding threshold $\hat{\theta}_{\mathrm{ph}}^{*}$. This, 
however, implies a higher likelihood of the actual loss amount being below the threshold value. As a consequence, the amount of fraud may increase up to the maximum of $20 \%$.

\section{Development of Net Present Value $N P V$}

Looking at Figure 5, it is striking that both $N P V$ and $\Delta U$ are positive for both chosen values of the cost per audit $k$. Especially with regard to gain in utility $\Delta U$, this implies that policyholders among the population are willing to adhere to the insurance relationship (see Equation (12)). This observation proves that the derived optimal auditing schemes are feasible from both stakeholders' perspectives.

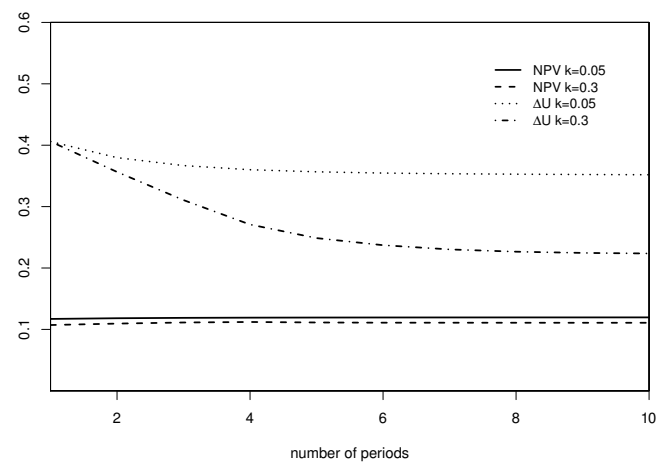

Figure 5: Development of the insurance company's net present value and policyholders' gain in utility, respectively, over the course of several iterations when applying the optimal auditing trategy for the respective period. The development is illustrated for two different choices of the cost per audit $k$. The remaining parameters are chosen as presented in Table 1.

From the insurance company point of view, higher costs per audit $k$ lead to a lower net present value $N P V$. The explanation is straightforward: higher auditing costs result in fewer auditing processes (see Figure 4(a)). Hence, we face a higher share of fraudulent claims (see Figure 4(b)). Clearly, more exaggerated claim amounts remain undetected, resulting in higher, unjustified expenses from the insurance company perspective.

Another intriguing result can be taken from Figure 5, recalling the case where behavioral adaptation is not possible. Since, in that simplified setting, signals are not exchanged, no changes in either the auditing or the claiming strategy would be possible, resulting in a stable solution after period two. Comparing the values from the second observation period with the subsequent ones in Figure 5, we see that, given the current setting, from the policyholder point of view, the gain in utility in the second observation period is considerably higher than the ones in all the subsequent observation periods, implying that the option to change one's strategy is disadvantageous to oneself. This result is very enlightening since it contradicts the widespread opinion that adapting the defrauding strategy based on signals, especially from third parties like service providers, is favorable from the individual's perspective. 


\subsubsection{Sensitivity Analyses}

The remainder of this section constitutes the presentation and discussion of the impact relevant input parameters have on the insurance company's optimal auditing strategy $\mathcal{A}_{\mathrm{R}}$ and the resulting effects on the net present value $N P V$. In particular, the influence of the cost per audit $k$, the relative fraud amount $\alpha$ and the policyholder's initial threshold value $\hat{\theta}_{\mathrm{ph}}^{*}$ will be analyzed.

For this purpose, we consider the final values of the optimal auditing range $\mathcal{A}_{\mathrm{R}}$, net present value $N P V$ and gain in utility $\Delta U$ after 12 iterations each when stable results are achieved.

\section{Cost Per Audit}

We take $k \in[0.05,0.5]$ and illustrate the results for the auditing range as well as the corresponding values for the insurance company's net present value $N P V$ in Figure 6 keeping the remaining parameters as presented in Table 1.

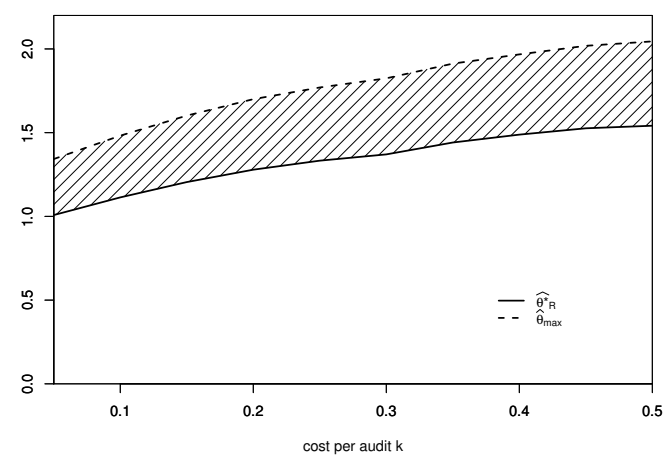

(a) Auditing range depending on cost per audit $k$

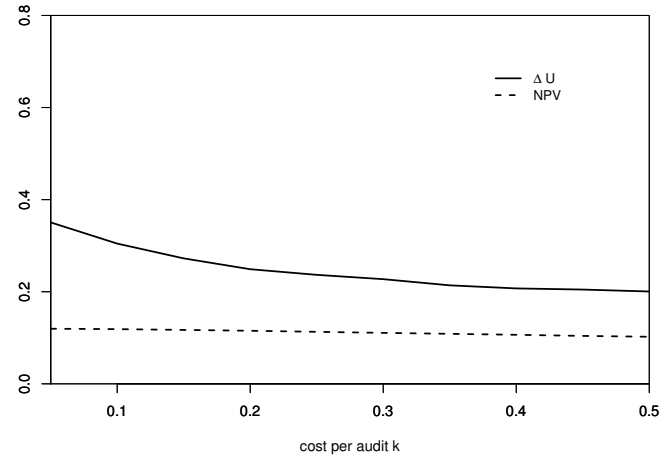

(b) Corresponding values for $\Delta U$ and $N P V$

Figure 6: Auditing range and the corresponding objective quantities from insurance company and policyholder depending on the cost per audit $k$. The remaining parameters are chosen as presented in Table 1.

Figure 6(a) shows that a change in the cost per audit $k$ merely has an impact on the width of the auditing range. However, the values themselves which trigger the verification process shift upwards for higher values of this specific input parameter. The higher the cost per audit $k$ is, the higher are the claim amounts which will be subject to auditing. These findings are in line with the ones presented in Figure 3. The lower the costs per audit are, the more verification processes the insurance company can perform assuming a given budget. This allows the insurer to review a larger number of incoming claims and consequently enhances the probability of revealing the fraudulent ones, in particular those which are close to the policyholder's threshold value. Such an approach enables the insurance company to adjust its auditing strategy optimally to the prevalent defrauding behavior at an early stage. As a consequence, potential escalations with regard to the fraud strategies, i.e., expansion of the policyholder's individual threshold value, can be prevented. In this context, note that the upper bound of the audit range in 
Figure 6 , which is defined by the maximum values of detected fraud, decreases when lowering the costs per audit $k$.

From the policyholder perspective, we observe that the gain in utility $\Delta U$ is always positive, implying that the auditing strategies discussed above are feasible.

\section{Relative Fraud Amount}

Considering $\alpha \in[0.25,2.5]$, we illustrate the effects on the optimal auditing strategy $\mathcal{A}_{\mathrm{R}}$ in Figure 7 and discuss them afterwards. Again, the remaining input parameters are chosen as given in Table 1.

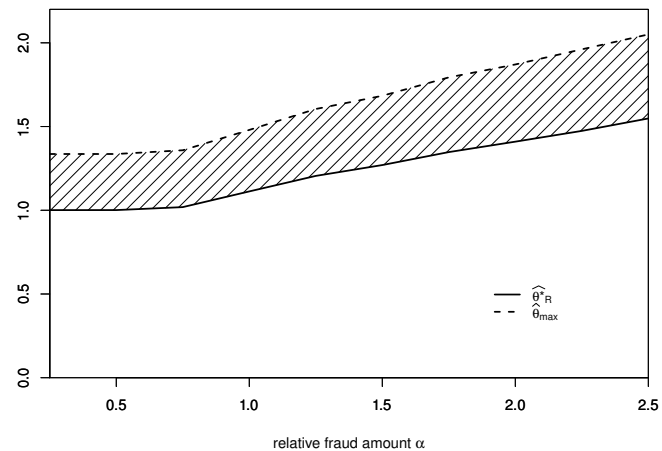

(a) Auditing range depending relative fraud amount $\alpha$

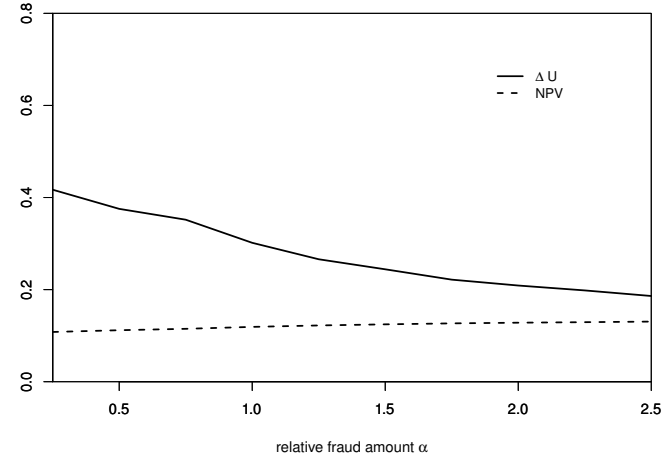

(b) Corresponding values for $\Delta U$ and $N P V$

Figure 7: Auditing range and the corresponding objective quantities from insurance company and policyholder depending on the relative fraud amount $\alpha$. The remaining parameters are chosen as presented in Table 1.

As can be seen in Figure 7(a), the relative fraud amount $\alpha$ has a considerable impact on the insurance company's optimal auditing strategy. The width of the auditing range increases (slightly) for greater values of this input parameter. At the same time, the claim values which indicate the need for verification shift in an upward direction. As a result, the relative fraud amount $\alpha$ also has an impact on both stakeholders' objective quantities. Figure 7 illustrates that the insurance company's net present value $N P V$ increases when the values of this input parameter are increased. The reason for this observation is that in case of a successful verification process, i.e., the detection of fraudulent behavior, the relative fraud amount $\alpha$ will become known. The higher the relative fraud amount $\alpha$ is - while assuming the loss distribution itself has not changed - the more profitable it is from the insurance company perspective to audit claims which demand high indemnity payments. Hence, the relative fraud amount $\alpha$ has a direct influence on the upper bound of the auditing range. This observation becomes even clearer when keeping in mind that the latter is determined by the maximum of all detected fraudulent claims during one period.

Furthermore, Figure 7(b) shows that the insurance company profits from raising and widening the auditing range whenever the relative fraud amount $\alpha$ is increased. In this current scenario, the relative fraud amount $\alpha$ is raised while keeping the probability for fraudulent behavior $p$ constant, i.e., fraud is not committed more often but more severely. Since the loss distribution remains unchanged, this implies that 
fraudulent claims are more likely to be those of higher value. From the insurance company perspective, this means that auditing becomes more profitable when shifting its auditing range into this area. Since in the case of detected fraudulent behavior no indemnity payments to the policyholder are made, this auditing strategy has a positive effect on the insurance company's net present value $N P V$.

Again, we see that the policyholder's gain in utility $\Delta U$ is positive throughout all observation periods, ensuring that the corresponding auditing strategies are feasible.

\section{Policyholder's Initial Threshold}

The final input parameter whose influence on the insurance company's optimal auditing strategy we aim to analyze is the policyholder's initial threshold value $\hat{\theta}_{\mathrm{ph}}^{*}$. This value serves as an upper bound for the potential fraud amount. As already introduced in Section 3.1, policyholders who decide to commit fraud exaggerate their loss amount by some constant factor $\alpha$ up to that threshold value $\hat{\theta}_{\mathrm{ph}}^{*}$. For the purpose of our sensitivity analysis, we consider $\hat{\theta}_{\mathrm{ph}}^{*} \in[1.05,1.5]$ and present the results in Figure 8 .

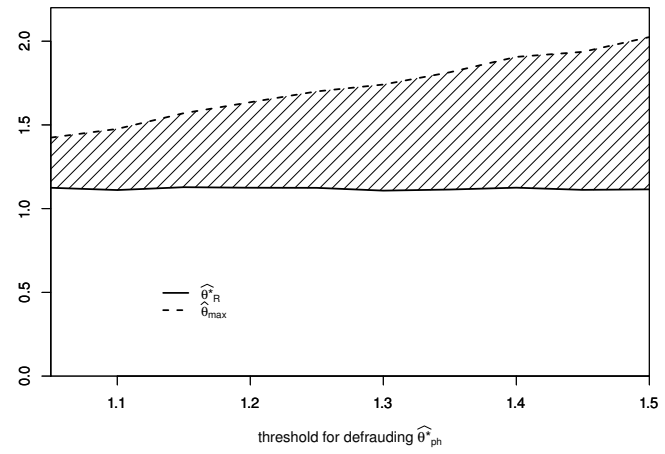

(a) Auditing range depending on policyholder's initial threshold $\hat{\theta}_{\mathrm{ph}}^{*}$

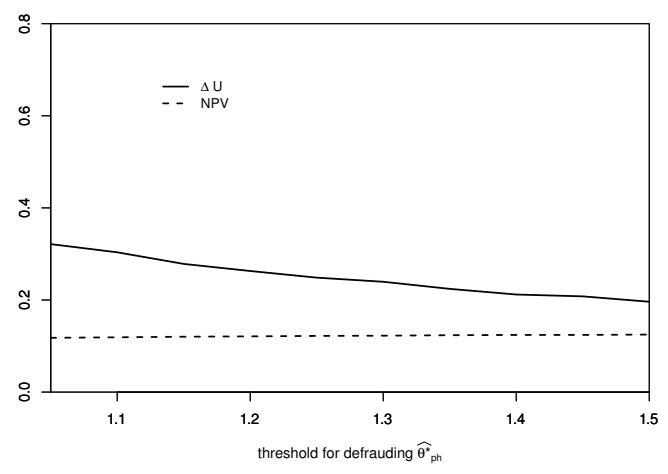

(b) Corresponding values for $\Delta U$ and $N P V$

Figure 8: Auditing range and the corresponding objective quantities from insurance company and policyholder depending on the policyholder's initial threshold value $\hat{\theta}_{\mathrm{ph}}^{*}$. The remaining parameters are chosen as presented in Table 1.

Figure 8 illustrates that the choice of policyholders' threshold value for defrauding $\hat{\theta}_{\mathrm{ph}}^{*}$ has a significant impact on the optimal auditing range $\mathcal{A}_{\mathrm{R}}$. Higher values of $\hat{\theta}_{\mathrm{ph}}^{*}$ imply an increasing discrepancy with regard to the insurance company's initial threshold for auditing $\hat{\theta}_{\text {init }}^{*}$ which is kept at a constant value of 1.0. As a result, the optimal auditing range $\mathcal{A}_{\mathrm{R}}$ becomes broader for increasing values of $\hat{\theta}_{\mathrm{ph}}^{*}$. In particular, the upper bound of the auditing range increases almost continuously while the lower bound remains almost constant. The explanation for this phenomenon is that an increase in policyholders' defrauding threshold $\hat{\theta}_{\mathrm{ph}}^{*}$ results in an increase in the share of fraudulent claims among all filed claims. In particular, the amount of exaggerated claims among those of higher value will increase. Since the upper bound of the optimal auditing range is determined as the maximum of detected fraud $\hat{\theta}_{\max }$ and some safety margin $s$, its value will become higher as well. 
This explanation can also be used for understanding the resulting marginal increase in the insurance company's net present value $N P V$. Since higher values of $\hat{\theta}_{\mathrm{ph}}^{*}$ lead to an increased likelihood of a loss amount below this threshold, the number of cases where fraud-prone policyholders engage in buildup increases, especially in the high-value segment. Whether this development has an impact on the insurance company's net present value depends on the prevalent auditing strategy $\mathcal{A}_{\mathrm{R}}$ and its detection success. Interpreting Figure 8(b), the number of detected fraudulent activities increases compared to the number of unjustifiably paid out claims resulting in fewer indemnification, which in turn leads to a slightly higher net present value.

As in the previous analyses, the gain in utility $\Delta U$ from having signed an insurance contract prior to the occurrence of loss is always positive implying that all participation constraints are met.

\section{Discussion of Results and Practical Implementations}

The insurance company's optimal auditing strategies as derived in this paper are characterized by two threshold values indicating the range of claimed values which should be subject to verification. All other incoming claims are indemnified without further specific proof of their accuracy. In particular, the approach presented here results in an examination of claims particularly from the medium-size segment, leaving out those claims of low and high values. This strategy is based on the assumption that policyholders avoid any engagement in fraudulent activities whenever the actual loss amount is above some personal threshold since they consider the possibility of being caught to be particularly high in this segment. As a consequence, theoretically there is no need to verify incoming claims of a higher magnitude.

From a practical point of view, however, this approach appears to be incomplete. It seems unimaginable that insurance companies will indemnify loss amounts which are far above the corresponding expected value without further examination of their legitimacy. For this purpose, we once more extend our model framework to accommodate this aspect. We therefore introduce an additional threshold value for auditing, $\hat{\theta}_{\text {high }}^{*}$. In addition to verifying all incoming claims whose values fall within the auditing range $\mathcal{A}_{\mathrm{R}}$, the insurance company also audits those which are above the new threshold value $\hat{\theta}_{\text {high }}^{*}$.

In order to depict the impact of adding an additional threshold value to the existing auditing range $\mathcal{A}_{\mathrm{R}}$, we consider $\hat{\theta}_{\text {high }}^{*}=1.5$, which equals one and a half times the expected value of the loss amount $\theta$. The remaining parameters are chosen as in the reference setting. Figure 9 illustrates the results.

As can be seen from Figure 9, in the particular setting of our model framework, an additional auditing threshold identifying high-value claims for verification does not generate benefit for the insurance company. The optimal auditing range $\mathcal{A}_{\mathrm{R}}$ including the additional threshold $\hat{\theta}_{\text {high }}^{*}$ results in a net present value of $N P V=0.118$ after the tenth observation period, whereas the optimal auditing range $\mathcal{A}_{\mathrm{R}}$ alone in $N P V=0.120$, i.e., we obtain a change of $2 \%$. From the policyholder perspective, the introduction of the new threshold value $\hat{\theta}_{\text {high }}^{*}$ has no impact. The gain in utility $\Delta U$ remains positive.

This observation can be explained by the underlying defrauding behavior of policyholders. Since fraud-prone individuals do not inflate loss amounts in this segment, the insurer performs costly auditing processes without ever detecting any fraudulent activities, i.e., additional costs arise without ever leading to savings due to refusal of indemnification. As a consequence, the insurance company's net present value 


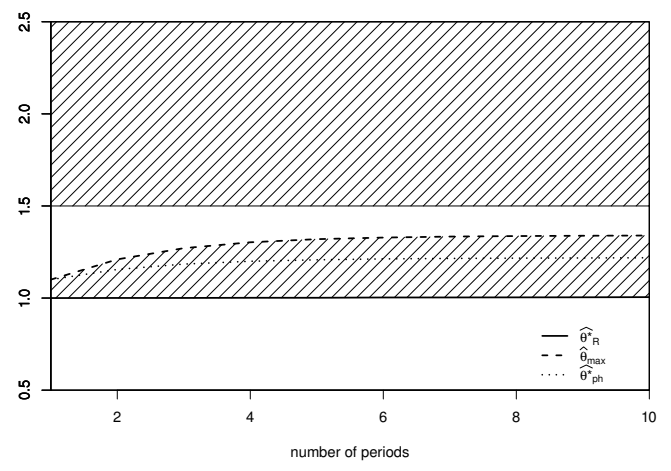

(a) Auditing range depending with additional auditing threshold $\hat{\theta}_{\text {high }}^{*}=1.5$

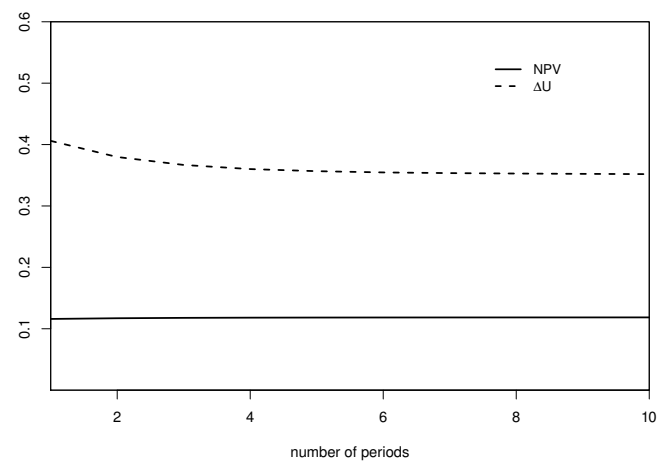

(b) Corresponding values for $\Delta U$ and $N P V$

Figure 9: Auditing range including an additional auditing threshold $\hat{\theta}_{\text {high }}^{*}=1.5$ and the corresponding objective quantities for insurance company and policyholder. The remaining parameters are chosen as presented in Table 1.

$N P V$ decreases when introducing the additional threshold value for auditing high-value claims. From the policyholder perspective, no changes in the gain in utility arise since the new auditing scheme has no impact on indemnification payments.

In practice, however, this observation does not necessarily have to hold true. On the one hand, policyholders and/or service providers might indeed engage in fraudulent activities in case of high-value losses or even if no insured loss occurred at all. As an example, Emerson (1992) reports the case of 'State vs. Book' in which the policyholder exaggerated the value of his stolen luxury class automobile by $20 \% .{ }^{6}$ On the other hand, insurance companies might profit from performing verification processes even in those cases when no fraudulent activities are detected. Their investigations might have a deterrent effect, discouraging policyholders and service providers trying to submit fraudulent claims in the future. The corresponding monetary benefits, however, are almost impossible to measure (see, e.g., Viaene and Dedene (2004)).

So far, we have assumed that when policyholders defraud, the consequence of detected fraud is a denial of indemnification. Even if this is often a contractual procedure in case of fraudulent policyholder behavior, in reality insurance companies may decide or are judicially required to pay the actual loss $\theta$ or a part of it, for example, in order to cover auditing expenses or to impose a penalty. This has been formalized, e.g., by Picard and Fagart (1999) through the introduction of a net indemnification $(\theta-B)$

\footnotetext{
${ }^{6}$ If the hypothesis that policyholders are not committing fraud above a specific claims amount is falsified via empirical observations or the insurer is afraid that policyholders may find out that large claims are not screened, the introduction of an additional upper threshold value as shown in this section would be necessary. The optimization procedure then provides an optimal auditing range as well as an optimal upper threshold value.
} 
where $B$ denotes the imposed penalty. With such notation, the contribution margin $C M$ for a single contract stated in Equation (5) can be rewritten as follows:

$$
C M(P, k, \hat{\theta}, \mathcal{A}, \mathcal{F})=P-\hat{\theta}\left[1-\mathbb{1}_{\mathcal{A} \cap \mathcal{F}}(\hat{\theta})\right]-(\theta-B) \cdot \mathbb{1}_{\mathcal{A} \cap \mathcal{F}}(\hat{\theta})-k \cdot \mathbb{1}_{\mathcal{A}}(\hat{\theta}) .
$$

The policyholder wealth position at the end of the observation period in time $t_{1}$ (compare with Equation (8)) becomes:

$$
W_{1}^{I}=W-P-\theta+\hat{\theta}\left[1-\mathbb{1}_{\mathcal{A} \cap \mathcal{F}}(\hat{\theta})\right]+(\theta-B) \cdot \mathbb{1}_{\mathcal{A} \cap \mathcal{F}}(\hat{\theta}) .
$$

Setting the penalty $B=0$, we present in Figure 10 the development of the optimal auditing range $\mathcal{A}_{\mathrm{R}}$ when the insurer's indemnification equals the actual loss in cases where a fraudulent policyholder claim $\hat{\theta}>\theta$ has been detected. Both graphs in Figure 10 provide information about the auditing strategy when costs per audit $k$ equal 0.05 and 0.3 in the reference case (parameterization from Table 1) are to be compared with the results in Figure 3 (Section 4.2.1). As expected, the results show that the optimal auditing range shifts to higher-valued claims. For example, at period $10, \hat{\theta}_{\mathrm{R}}^{*}$ yields $+11 \%$ (1.11 instead of 1.00) for $k=0.05$ and $+16 \%$ (1.61 instead of 1.39) for $k=0.3$ when paying the actual loss instead of denying any payment. This gives a good impression of sensitivity when using various payment guidelines for fraudulent claims.

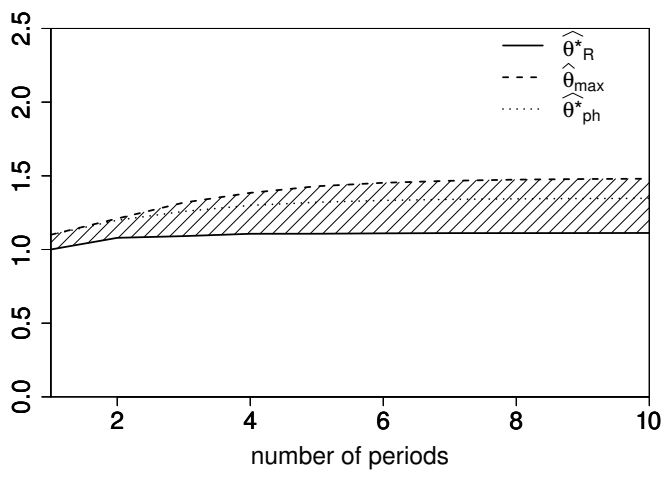

(a) Development of optimal auditing strategy, $k=0.05$

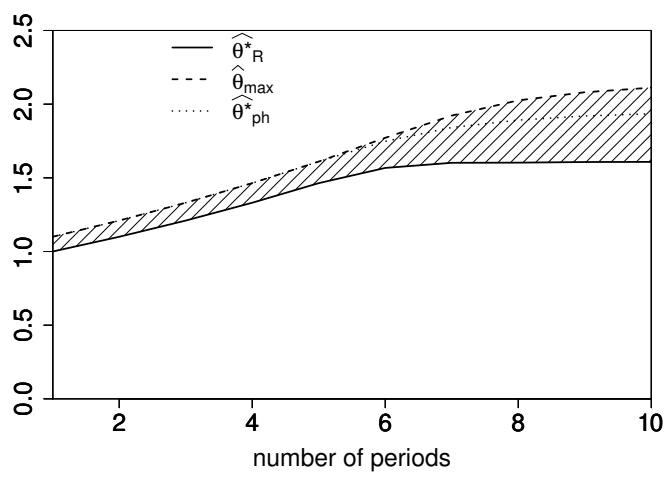

(b) Development of optimal auditing strategy, $k=0.3$

Figure 10: Development of the optimal auditing strategy when the actual loss $\theta$ is paid for fraudulent claims $\hat{\theta}>\theta$ (see Equations (24) and (25)) throughout the course of several iterations for two different choices of the cost per audit $k$. The remaining parameters are chosen as presented in Table 1.

The quantitative target function used to measure policyholders' utility only valuates future cash flows. However, several aspects that play a role in real-life behavior are omitted in our model. For instance, honest policyholders who have been investigated by the insurer may find this procedure to be a negative experience and hence switch to another provider. Policyholders that have been caught (and maybe sued by the insurer) when committing an insurance fraud may experience this as an embarrassing event and - in addition to the actions performed by the insurer - typically face difficulties in getting insurance 
coverage in the future. In order to account for these effects, empirical information is needed to estimate the influence of those factors on the utility of the policyholders in the insurer's portfolio.

However, some cases of such 'negative experience' could be formally integrated in our model setup. In our basic setting, $80 \%$ of the total number of policyholders (2500 000) do not commit insurance fraud. For most auditing strategies, some of the honest policyholders will be reviewed. Hence, we could derive an optimal auditing strategy assuming that some positive percentage of the nonfraudulent policyholders would cancel the contract in $t=1$ whenever an examination took place. The effects of such a behavior by some policyholders are quite straightforward: in the optimal auditing strategy, the insurer would perform less auditing compared to the situation without contract surrender. The outcome is comparable to the case where the costs per auditing $k$ are increased (see Section 4.2.1).

In addition, we need to clarify that our modeling of a behavioral adaption is restricted to cases that are certainly not always fulfilled in insurance practice. If only two parties are involved - the insurer and the insured - we assume that fraud-prone policyholders are interested in identifying the insurer's auditing range (defined in monetary units). However, committing insurance fraud may not be triggered primarily by this kind of consideration. If a third party is involved - for instance a repair shop in case of motor insurance we assumed that fraudulent activities, even if committed by repair shops, are advantageous for policyholders only. The incentive for a repair shop to engage in fraudulent activities against the insurance company may stem from the hope of gaining the policyholder's favor and thereby keeping him as a customer in the future. Certainly, many other motives play a role in practice. For instance, the repair shop may commit fraud without the knowledge of the policyholder. Such a case is only covered in our model if we assume that the decisions made by the repair shop are based on the line of reasoning laid down in Section 2.3 (maximizing expected utility) and repair shops are not punished when providing a 'built-up' strategy. With regard to the last point, it must be remembered that insurance companies provide business for specific approved repair stations. These repair stations may lose their status when a building-up of claims is discovered. Hence, there may be a trade-off decision for the repair station. As a result, the repair station is not interested in buildup strategies in the insurer's auditing range. In such a situation, the behavioral adaption reduces to a pure static result based on a two-points-in-time model.

\section{Conclusion}

In this paper we develop a model framework which depicts an optimal auditing scheme from the viewpoint of an insurance company with regard to inflated insurance claims. In the model setup provided in this paper, the insurance company maximizes its profits while at the same time maintaining contract attractiveness such that policyholders are willing to adhere to the insurance relationship and hence purchase insurance coverage. The central outcome of our model is an auditing range which selects those claims which should be subject to verification. This auditing range is triggered by the filed amount.

Using a numerical approach based on Monte Carlo simulations, we are able to illustrate and analyze the impact of different parameterizations on the optimal auditing range in form of a sensitivity analysis. High costs per audit as well as a high relative fraud amount result in an upward shift of the auditing range, whereas an increase in policyholders' defrauding threshold leads to a broadening of the range. 
With regard to the insurance company's objective function used in the model, the relative fraud amount has a particularly strong impact on its result.

In this context, we take account of the ability of each stakeholder to adapt its behavioral strategy over the course of several periods. By this means, we take into consideration that changes in policyholder defrauding behavior can have a crucial impact on the optimal auditing strategy and vice versa. Insurance companies can use their experiences from previous verification processes as a source of information whereas policyholders may rely on third parties (and independent contractors).

When calculating the optimal auditing range from the insurer's point of view, we come to the conclusion that given some constant cost per audit it is optimal to verify the accuracy of claims from the mid-value segment. In particular, it is not reasonable for the insurer to examine small claims since the accompanying costs outweigh the savings potential in case of detected fraud. Not verifying high-value claims results from the assumption that fraud-prone policyholders do not inflate the magnitude of their losses above some personal threshold value since they fear that the likelihood of getting caught in this segment to be high. Omitting this assumption, however, may require the introduction of an additional threshold value for auditing. Furthermore, we are able to show that while the option to adapt one's strategy might be favorable from the insurance company perspective, it has a negative impact on the policyholders' position compared to the situation where no signals are exchanged based on which one could adapt one's behavior. This result disproves the common belief that adapting the defrauding strategy with the help of signals from service providers (and independent contractors) would be advantageous from the policyholder point of view.

\section{References}

Bearing Point, 2008, Fraud Management - Trends in Insurance, Technical Report.

Belhadji, B., G. Dionne, and F. Tarkhani, 2000, A Model for the Detection of Insurance Fraud, Geneva Papers on Risk and Insurance - Issues and Practice, 25(4):517-538.

Bermúdez, L., J. Pérez, M. Ayuso, E. Gómez, and F. Vázquez, 2008, A Bayesian Dichotomous Model with Asymmetric Link for Fraud in Insurance, Insurance: Mathematics and Economics, 42(2):779-786.

Bond, E. and K. Crocker, 1997, Hardball and the Soft Touch: The Economics of Optimal Insurance Contracts with Costly State Verification and Endogenous Monitoring Costs, Journal of Public Economics, 63(2):239-264.

Crocker, K. and J. Morgan, 1998, Is Honesty the Best Policy? Curtailing Insurance Fraud through Optimal Incentive Contracts, Journal of Political Economy, 106(2):355-375.

Crocker, K. and S. Tennyson, 2002, Insurance Fraud and Optimal Claims Settlement Strategies, Journal of Law and Economics, 45(2):469-507.

Cummins, D. and O. Mahul, 2004, The Demand for Insurance with an Upper Limit on Coverage, Journal of Risk and Insurance, 71(2):253-264.

Derrig, R., 2002, Insurance Fraud, Journal of Risk and Insurance, 69(3):271-287. 
Derrig, R. and V. Zicko, 2002, Prosecuting Insurance Fraud - A Case Study of the Massachusetts Experience in the 1990s, Risk Management and Insurance Review, 5(2):77-104.

Dionne, G. and O. Ghali, 2005, The (1992) Bonus-Malus System In Tunisia: An Empirical Evaluation, Journal of Risk and Insurance, 72(4):609-633.

Dionne, G., F. Giuliano, and P. Picard, 2009, Optimal Auditing with Scoring: Theory and Application to Insurance Fraud, Management Science, 55(1):58-70.

Dionne, G. and C. Vanasse, 1992, Automobile Insurance Ratemaking In The Presence Of Asymmetrical Information, Journal of Applied Econometrics, 7(2):149-165.

Duffield, G. and P. Grabosky, 2001, The Psychology of Fraud, In Trends and Issues in Crime and Criminal Justice, 199. Australian Institute of Criminology.

Dulleck, U. and R. Kerschbamer, 2006, On Doctors, Mechanics, and Computer Specialists: The Economic of Credence Goods, Journal of Economic Literature, 44(1):5-42.

Emerson, R., 1992, Insurance Claims Fraud Problems and Remedies, University Of Miami Law Review, 46:907-973.

GDV, 2011, Versicherungsbetrug: aktuelle Entwicklungen, Muster und ihre Abwehr, Technical Report.

Hubbard, T., 2002, How Do Consumers Motivate Experts? Reputational Incentives inan Auto Repair Market, Journal of Law and Economics, 45(2):437-468.

Insurance Research Council, 2008, Fraud and Buildup in Auto Injury Insurance Claims, Technical Report.

Kahneman, D. and A. Tversky, 1979, Prospect Theory: An Analysis of Decision under Risk, Econometrica, $47(2): 263-292$.

Kerr, D., 2012, Exploring the Role of Pseudodeductibles in Auto Insurance Claims Reporting, Journal of Insurance Issues, 35(1):44-72.

Lacker, J. and J. Weinberg, 1989, Optimal Contracts under Costly State Falsification, Journal of Political Economy, 97(6):1345-1363.

Mahlow, N., S. C. Maier, P. Müller, J. Schmidt, and J. Wagner, 2015, Trends im Schadenmanagement 2015 - Digitalisieung, Betrugsbekämpfung, Dienstleistermanagement, Technical Report, HEC Lausanne \& Innovalue Management Advisors.

Marlin, P., 1984, Fitting the Log-Normal Distribution to Loss Data Subject to Multiple Deductibles, Journal of Risk and Insurance, 51(4):627-701.

Miyazaki, A. D., 2008, Perceived Ethicality of Insurance Claim Fraud: Do Higher Deductibles Lead to Lower Ethical Standards?, Journal of Business Ethics, 87(4):589-598.

Mookherjee, D. and I. Png, 1989, Optimal Auditing, Insurance, and Redistribution, Quarterly Journal of Economics, 104(2):399-415.

Moreno, I., F. Vázquez, and R. Watt, 2006, Can Bonus-Malus Alleviate Insurance Fraud?, Journal of Risk and Insurance, 73(1):123-151.

Morley, N., L. Ball, and T. Ormerod, 2006, How the detection of insurance fraud succeeds and fails, Psychology, Crime \& Law, 12(2):163-180. 
Müller, K., 2014, The Identification of Insurance Fraud - An Empirical Analysis, I.VW-HSG Working Paper.

Picard, P., 2000, On the Design of Optimal Insurance Policies Under Manipulation of Audit Cost, International Economic Review, 41(4):1049-1071.

Picard, P., 2001, Economic Analysis of Insurance Fraud, In Handbook of Insurance, 1997. Springer.

Picard, P. and M.-C. Fagart, 1999, Optimal Insurance Under Random Auditing, Geneva Papers on Risk and Insurance Theory, 24(1):29-54.

Tennyson, S., 2008, Moral, Social, and Economic Dimensions of Insurance Claims Fraud, Social Research, $74(4): 1181-1204$.

Townsend, R., 1979, Optimal Contracts and Competitive Markets with Costly State Verification, Journal of Economic Theory, 21(2):265-293.

Viaene, S., M. Ayuso, M. Guillen, D. Van Gheel, and G. Dedene, 2007, Strategies for detecting fraudulent claims in the automobile insurance industry, European Journal of Operational Research, 176:565-583.

Viaene, S. and G. Dedene, 2004, Insurance Fraud: Issues and Challenges, Geneva Papers on Risk and Insurance - Issues and Practice, 29(2):313-333.

Viaene, S., R. A. Derrig, B. Baesens, and G. Dedene, 2002, A Comparison of State-of-the-Art Classification Techniques for Expert Automobile Insurance Claim Fraud Detection, The Journal of Risk and Insurance, 69(3):373-421.

Watt, R., 2003, Curtailing Ex-Post Fraud in Risk Sharing Arrangements, European Journal of Law and Economics, 16(2):247-263.

Weisberg, H. and R. Derrig, 1991, Fraud and Automobile Insurance: A Report on Bodily Injury Liability Claims in Massachusetts, Journal of Insurance Regulation, 9(4):497-541. 